\title{
Forced Responses of the Parametric Vibration System for the Electromechanical Integrated Magnetic Gear
}

\author{
Xiu-hong Hao ${ }^{1}$ and Xue-jun $\mathrm{Zhu}^{2,3}$ \\ ${ }^{1}$ School of Mechanical Engineering, Yanshan University, Qinhuangdao 066004, China \\ ${ }^{2}$ Key Laboratory of Advanced Forging \& Stamping Technology and Science, Yanshan University, Ministry of Education of China, \\ Qinhuangdao 066004, China \\ ${ }^{3}$ Hebei Provincial Key Laboratory of Parallel Robot and Mechatronic System, Yanshan University, Qinhuangdao 066004, China \\ Correspondence should be addressed to Xiu-hong Hao; hxhong@ysu.edu.cn
}

Received 22 March 2015; Revised 18 June 2015; Accepted 28 June 2015

Academic Editor: Laurent Mevel

Copyright (C) 2015 X.-h. Hao and X.-j. Zhu. This is an open access article distributed under the Creative Commons Attribution License, which permits unrestricted use, distribution, and reproduction in any medium, provided the original work is properly cited.

Considering the magnetic fields modulating in the electromechanical integrated magnetic gear (EIMG), the electromagnetic coupling stiffnesses vary periodically and the expressions are given by the finite element method. The parametric vibration model and the dynamic differential equations are founded. The expressions of forced responses of EIMG system are deduced when the main resonances and the combination resonances occur. And then, the time and frequency responses are figured out. The dynamic characteristics of EIMG system are discussed. The results show that the dominant frequencies in the resonances are always the natural frequency of EIMG system. The relative amplitudes of the components have great difference and the components amplitudes of the main resonances are much bigger than the components amplitudes of the combination resonances. The time-varying meshing stiffness wave between the inner stator and the inner ferromagnetic pole-pieces has little influence on EIMG system.

\section{Introduction}

Since the high performance rare-earth permanent magnet materials came out in the 1980s, magnetic gears attracted much attention and were widely applied in industries because of their advantages, such as no mechanical fatigue, low noises, little maintenance, no lubrication, and inherent overload protection, while, because they adopt the parallel shaft topology, the traditional magnetic gears have small output torques and small torque density. So, the uses of the traditional magnetic gears are limited greatly [1].

Field modulated magnetic gear (FMMG) adopts the coaxial topology and significantly improves the utilization of the permanent magnets (PMs) [2]. Except for the advantages of the traditional magnetic gears, FMMG can provide bigger torque and higher torque density [1-3]. So, FMMG can be widely used in the medicine, chemical, vehicle, navigation, and other fields $[4,5]$. Based on the FMMG, the axial and linear field modulated magnetic gears were proposed
$[3,6]$. Many kinds of the direct current and alternating current permanent magnet motors were presented $[7,8]$. Transmission mechanism $[2,3]$, torque characteristics $[3,4$, 9], structural optimization [10], rotor eccentricity [11], and dynamics [12] have been discussed widely. The transmission performance of the FMMG system is improved greatly.

The coaxial topology makes FMMG readily be integrated with electric machinery. In this paper, a new kind of the electromechanical integrated magnetic gear (EIMG) is proposed by authors, in which FMMG, drive, and control are integrated. Compared with FMMG, EIMG has two stage transmissions. So, EIMG has a compact structure and makes the electromechanical drive system be simplified drastically. Meanwhile, the output torque and speed can be controlled. EIMG can offer a larger torque at a lower speed and can be applied in robot control, aerospace, navigation, vehicle, and other drive fields, in which high control precisions are required. 


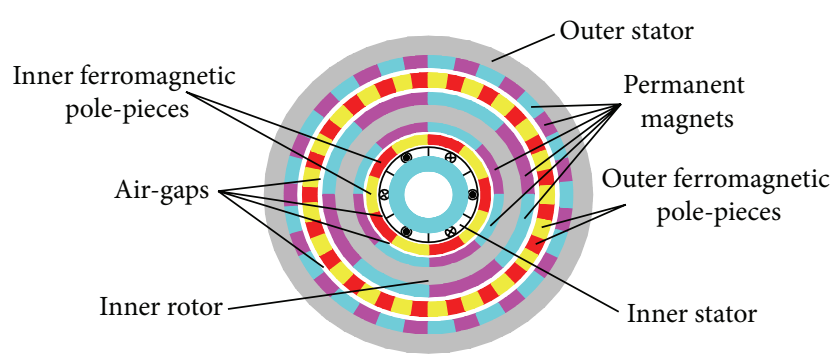

FIGURE 1: Topology and prototype of the electromechanical integrated magnetic gear.

During running of EIMG system, the ferromagnetic polepieces take charge of modulating the magnetic fields in two air-gaps beside them and make the electromagnetic coupling stiffnesses among components vary periodically. In mechanical gear systems, the meshing stiffnesses vary with rectangular waves because of the periodical change of the meshing tooth pair number [13]. However, the meshing number of pole pairs is always a constant and the increment components of the electromagnetic coupling stiffnesses vary with a cosine function. Considering the time-varying electromagnetic coupling stiffnesses, EIMG system belongs to a typical parametric vibration system. When there are some outer exciting sources, the main resonances and the combination resonances will occur. The resonances will worsen the dynamic characteristics of EIMG system and should be avoided.

In this paper, the finite element model of EIMG system is built and the expressions of the electromagnetic coupling stiffnesses are given. Considering the time-varying electromagnetic coupling stiffnesses, the parametric vibration dynamic model of EIMG system and the dynamic differential equations are founded. By the multiscale method, the approximate analytical solutions are deduced when the main resonances and the combination resonances occur. Then, the resonance responses are discussed and the results can provide the theoretical foundations for the parameter optimizations and the dynamic characteristics improvement of EIMG system.

\section{Parametric Vibration Model of EIMG System}

2.1. Electromagnetic Coupling Stiffnesses of EIMG System. EIMG shown in Figure 1 is mainly composed of the inner stator, the inner ferromagnetic pole-pieces (FPs), the inner rotor, the outer FP, and the outer stator. Three-phase coils are arranged in the inner stator and can generate a rotating magnetic field in order to provide power. PMs of the $\mathrm{N}$ and $S$ poles are arranged uniformly on the inner surface of the outer stator, the inner and outer surfaces of the inner rotor. The inner and outer FPs take charge of modulating the magnetic fields in two air-gaps beside them in order to make the number of pole pairs of the PMs agree with the number of pole pairs of the space harmonic flux density of the air-gaps.
The finite element model of the example EIMG system shown in Table 1 can be founded. Here, the material of the inner stator is silicon steel sheets. The back iron materials of the inner rotor and the outer stator are Q235 steel. The materials of the magnetic components and nonmagnetic components in FPs are 23TW250 and epikote, respectively. The properties of the epikote basically agree with air. Meshing is carried out manually and the rapid calculations can be realized by controlling the grid refinement level.

The meshes between the back iron and PMs of the inner rotor have common boundary nodes. During running of EIMG system, all unit nodes on PMs and the back iron of the inner rotor rotate together as a rigid body. Similarly, the back iron and PMs of the outer stator have common boundary nodes. The meshes on the magnetic components and nonmagnetic components of the inner and outer FPs have common boundary nodes, respectively. The middle positions of the air-gaps are not the common areas of the nodes. The magnetic field distribution is calculated by interpolations among the boundary nodes.

The reluctances of the air-gaps are bigger than PMs and the back irons. Meanwhile, the thicknesses of the air-gaps are smaller. So, the grid density in the air-gaps is bigger. Although there are some errors, the errors are negligible if the meshes of the air-gaps are very small. The initial grid model of the example EIMG system and the magnetic field distribution can be figured out in Figure 2.

The magnetic flux density in the middle of the air-gaps is $B_{j}$, where $j$ is the number of four air-gaps, $j=1,2,3,4$. $B_{\theta j}$ and $B_{r j}$ are the tangential and radial components of the magnetic flux density $B_{j}$, respectively. The tangential electromagnetic coupling forces among components can be calculated by Maxwell stress tensor method. Then, the electromagnetic coupling stiffnesses among components can be obtained as follows:

$$
k_{i t}=\frac{\mathrm{d} F_{i t}}{\mathrm{~d} u_{i t}}=\frac{\mathrm{d} F_{i t}}{\left(R_{j} \mathrm{~d} \theta\right)}=\frac{\left(\left(L / \mu_{0}\right) \int_{0}^{2 \pi} B_{\theta j} B_{r j} \mathrm{~d} \theta\right)}{\mathrm{d} \theta},
$$

where $F_{i t}$ and $k_{i t}, i=$ Is, II, Io, oo, are the tangential components of the electromagnetic coupling forces and the electromagnetic coupling stiffnesses between the inner stator and the inner FP, the inner FP and the inner rotor, the inner rotor and the outer FP, and the outer FP and the outer stator, respectively; $L$ is the axial effective length of EIMG system; $R_{j}$ is the radius of the middle position of the air-gap among components; $\mu_{0}$ is the space permeability; $\theta$ is the mechanical rotation angle.

When the coils in the inner stator are connected to the three-phase alternating currents, the number match of the pole pairs of the PMs is realized by the inner and outer FPs modulating the magnetic fields, while, because of the magnetic fields modulating, the electromagnetic coupling stiffnesses among components vary periodically. The dynamic electromagnetic coupling stiffnesses can be worked out by the finite element method and shown in Figure 3 .

Figure 3 shows that the stiffnesses fluctuations between the inner stator and the inner FP, the inner rotor and the outer FP, are bigger than others and cannot be ignored. 
TABLE 1: Parameters of example EIMG system.

\begin{tabular}{lcl}
\hline Number of pole pairs on the inner surface of the inner rotor & 5 & Number of pole pairs on the inner stator \\
Number of pole pairs on the outer surface of the inner rotor & 4 & Number of pole pairs on the outer stator \\
Number of the inner ferromagnetic pole-pieces & 7 & Number of the outer ferromagnetic pole-pieces \\
Outer radius of the inner stator/mm & 98 & Inner radius of the inner FP/mm \\
Outer radius of the inner FP/mm & 130 & Thickness of PMs on the inner rotor/mm \\
Inner radius of the inner rotor yoke/mm & 152 & Outer radius of the inner rotor yoke/mm \\
Inner radius of the outer FP/mm & 204 & Outer radius of the outer FP/mm \\
Thickness of PMs on the outer stator/mm & 10 & Inner radius of the outer stator yoke/mm \\
Outer radius of the outer stator yoke/mm & 276 & Axial length/mm \\
Remanence of PMs/T & 1.3 & Coercive force of PMs/KOe \\
Magnitude of currents on the inner stator/A & 20 & Current frequency/Hz \\
\hline
\end{tabular}

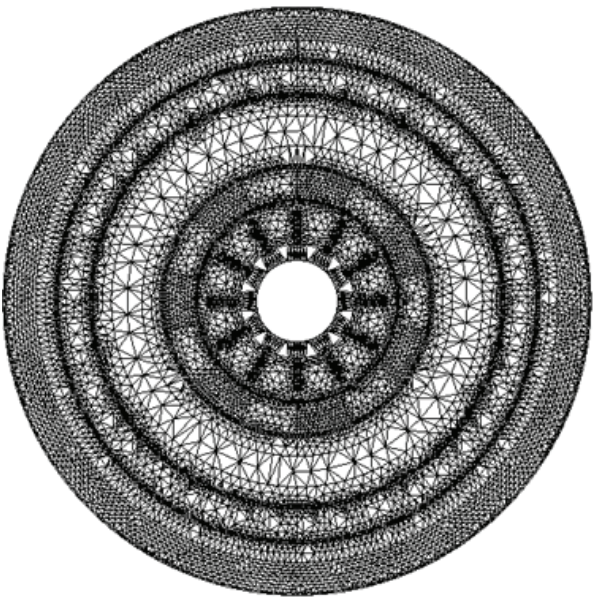

(a) Initial grid model

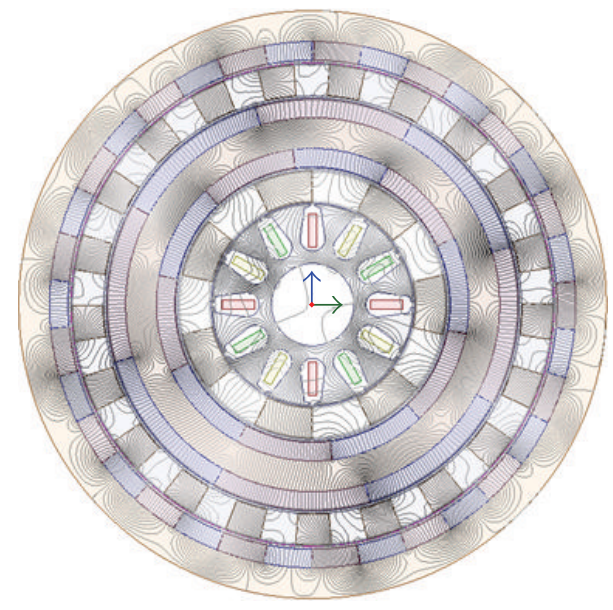

(b) Magnetic field distribution

Figure 2: Finite element model of the example EIMG system.

The dynamic electromagnetic coupling stiffnesses contain multiple harmonics. The main frequency of the stiffness fluctuation between the inner stator and the inner FP is the product of the current frequency and the number of pole pairs of the three-phase alternating currents on the inner stator. The main frequency of the stiffness fluctuation between the inner rotor and the outer FP is the product of the angular frequency and the number of pole pairs of the PMs on the outer surface of the inner rotor. Considering the amplitudes of other frequency components are much smaller, the time-varying electromagnetic coupling stiffnesses $k_{\mathrm{Is}}(t)$ and $k_{\mathrm{II}}(t)$ can be simplified as follows:

$$
\begin{aligned}
& k_{\mathrm{Is}}(t)=\bar{k}_{\mathrm{Is}}+2 \Delta k_{\mathrm{Is}} \cos \omega_{\mathrm{s}} t=\bar{k}_{\mathrm{Is}}\left(1+\varepsilon_{\mathrm{Is}} e^{\omega_{\mathrm{s}} t}\right)+\mathrm{cc}, \\
& k_{\mathrm{Io}}(t)=\bar{k}_{\mathrm{Io}}+2 \Delta k_{\mathrm{Io}} \cos \omega_{\mathrm{o}} t=\bar{k}_{\mathrm{Io}}\left(1+\varepsilon_{\mathrm{Io}} e^{\omega_{\mathrm{o}} t}\right)+\mathrm{cc},
\end{aligned}
$$

where $\bar{k}_{\text {Is }}$ and $\bar{k}_{\text {Io }}$ are the average values of the electromagnetic coupling stiffnesses $k_{\mathrm{Is}}(t)$ and $k_{\mathrm{Io}}(t)$, respectively; $\Delta k_{\mathrm{Is}}$ and $\Delta k_{\mathrm{Io}}$ are the increments of the stiffnesses $k_{\mathrm{Is}}(t)$ and $k_{\mathrm{Io}}(t)$, respectively; $\omega_{\mathrm{s}}$ and $\omega_{\mathrm{o}}$ are the fluctuation frequencies of the stiffness increments $\Delta k_{\mathrm{Is}}$ and $\Delta k_{\mathrm{IO}}$, respectively; namely, meshing frequencies $\omega_{\mathrm{s}}=2 \pi n_{\mathrm{s}} p_{\mathrm{s}} / 60, \omega_{\mathrm{o}}=2 \pi n_{\mathrm{I}} p_{\mathrm{I}} / 60 ; n_{\mathrm{s}}$ and $p_{\mathrm{s}}$ are the frequency and the pole pairs of the threephase alternating currents on the inner stator, respectively; $n_{\mathrm{I}}$ and $p_{\mathrm{I}}$ are the revolutions per minute and the pole pairs of PMs of the inner rotor, respectively; $\varepsilon_{\mathrm{Is}}$ and $\varepsilon_{\mathrm{IO}}$ are the small parameters of the electromagnetic coupling fluctuation, respectively, $\varepsilon_{\mathrm{Is}}=2 \Delta k_{\mathrm{Is}} / \bar{k}_{\mathrm{Is}}$ and $\varepsilon_{\mathrm{Io}}=2 \Delta k_{\mathrm{Io}} / \bar{k}_{\mathrm{Io}} ; \mathrm{cc}$ is the conjugate complex of the right expression in (2).

The values of small parameters $\varepsilon_{\mathrm{Is}}$ and $\varepsilon_{\mathrm{Io}}$ are 0.041 and 0.0355 , respectively. They are rather small and the numerical difference is smaller. Here, $\varepsilon_{\mathrm{IS}}$ and $\varepsilon_{\mathrm{IO}}$ are uniformly simplified with the same parameter $\varepsilon$ and $\varepsilon=0.04$.

\subsection{Parametric Vibration Model and Dynamic Differential} Equations. When the outer FP is fixed, the parametric vibration model of EIMG system shown in Figure 4 contains four subsystems, namely, the inner stator/inner FP subsystem, the inner FP/inner rotor subsystem, the inner rotor/outer FP subsystem, and the outer FP/outer stator subsystem. The model allows each part to rotate about their translation axes. 


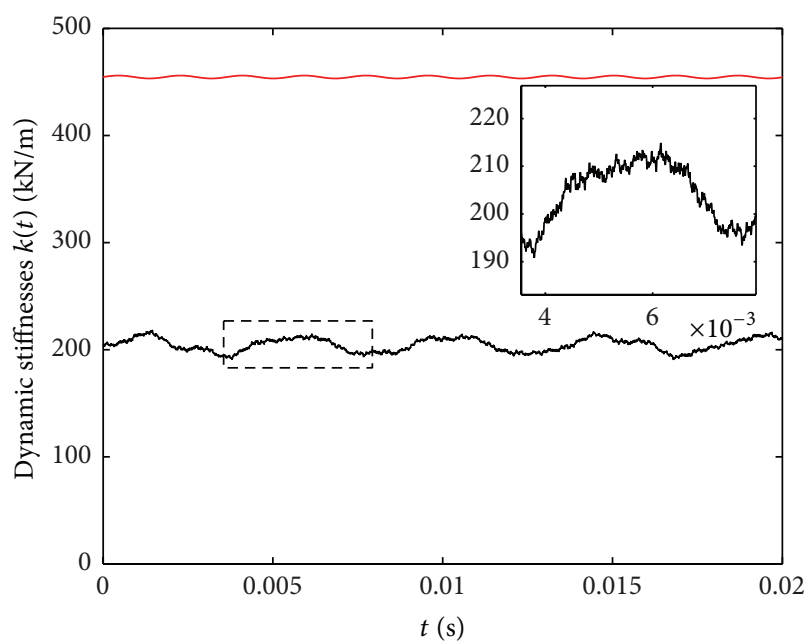

(a) $k_{\text {Is }}(t)$ and $k_{\text {II }}(t)$

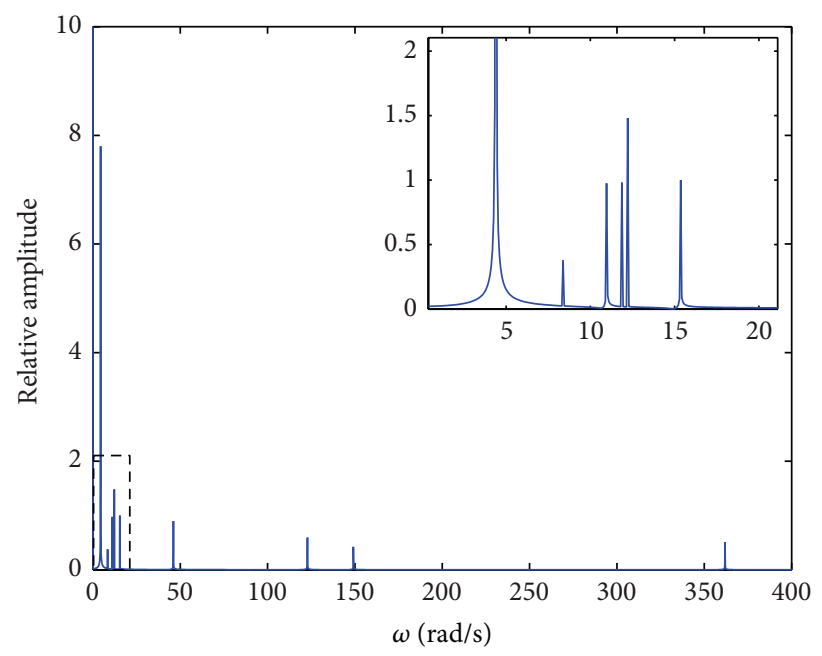

(c) Frequency component of $k_{\text {Is }}(t)$

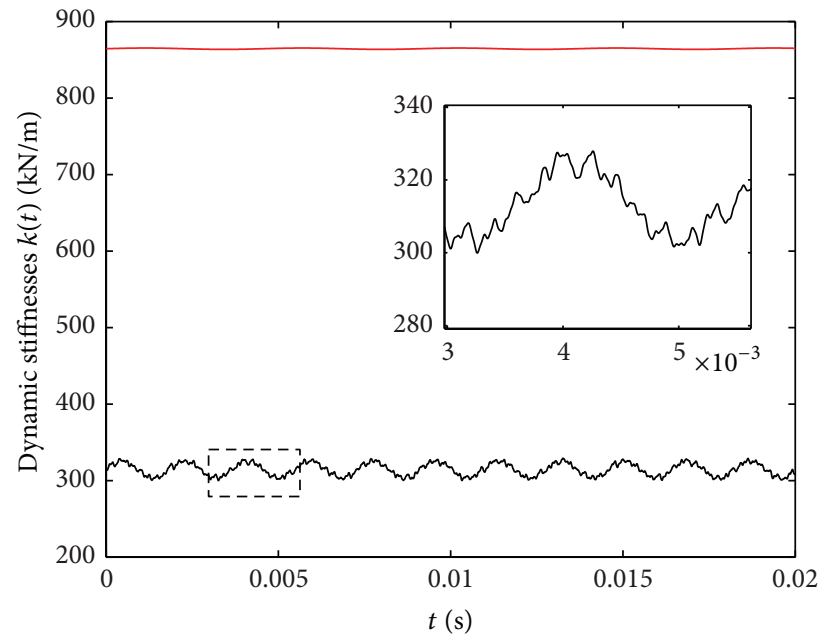

(b) $k_{\mathrm{Io}}(t)$ and $k_{\mathrm{oo}}(t)$

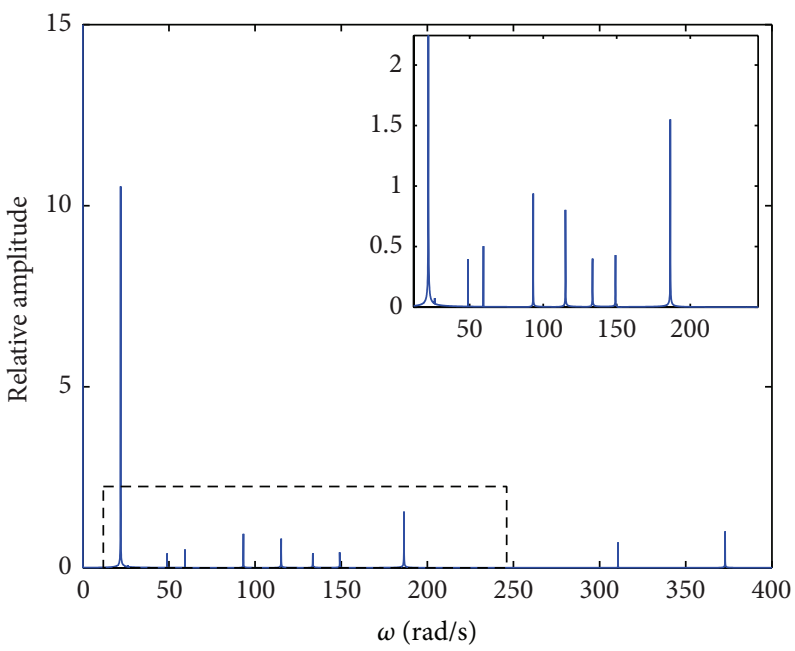

(d) Frequency component of $k_{\mathrm{Io}}(t)$

Figure 3: Dynamic electromagnetic coupling stiffnesses among components.

The electromagnetic coupling dynamic model employs the following assumptions:

(1) Main components of EIMG system are considered to be rigid, assuming that the elastic deformations of all the components are negligible.

(2) Constraints between the inner stator, the inner FP, the outer stator, and the foundation are equivalent to the tangential linear spring, respectively.

(3) Although EIMG system has the maximum output torque, overload does not occur. Manufacturing and installation errors of all components are not included in this paper.

(4) All PMs on the inner and outer surfaces of the inner rotor are assumed to be identical with the same size and performance parameters, respectively. All PMs on the outer stator have the same size and performance parameters too. Magnetic components and nonmagnetic components in the inner and outer FPs have the same sizes and performance parameters, respectively.

After defining the differential equations of individual subsystem, the differential equations of five degrees of freedom (DOF) of the overall EIMG system can be given in matrix form as

$$
\mathbf{M} \ddot{\mathbf{x}}+\mathbf{C} \dot{\mathbf{x}}+\mathbf{K x}=\mathbf{F}
$$

The mass matrix $\mathbf{M}$, the damping matrix $\mathbf{C}$, the stiffness matrix $\mathbf{K}$, the load vector $\mathbf{F}$, and the displacement vector $\mathbf{x}$ are 
derived as follows:

$$
\begin{aligned}
& \mathbf{M}=\operatorname{diag}\left(\left[\begin{array}{lllll}
M_{\mathrm{s}} & M_{\mathrm{If}} & M_{\mathrm{I}} & M_{\mathrm{of}} & M_{\mathrm{o}}
\end{array}\right]\right), \\
& \mathbf{C}=\operatorname{diag}\left(\left[\begin{array}{lllll}
c_{\mathrm{s}} & c_{\mathrm{If}} & c_{\mathrm{I}} & c_{\mathrm{of}} & c_{\mathrm{o}}
\end{array}\right]\right), \\
& \mathbf{F}=\left[\begin{array}{lllll}
0 & 0 & 0 & \frac{-\Delta T_{\text {of }} \cos \omega_{\mathrm{e}} t}{R_{\text {of }}} & 0
\end{array}\right]^{\mathrm{T}}, \\
& \mathbf{x}=\operatorname{diag}\left(\left[\begin{array}{lllll}
u_{\mathrm{s}} & u_{\mathrm{If}} & u_{\mathrm{I}} & u_{\mathrm{of}} & u_{\mathrm{o}}
\end{array}\right]\right), \\
& \mathbf{K}=\left[\begin{array}{ccccc}
\bar{k}_{\mathrm{Is}} \cos ^{2} \alpha_{\mathrm{Is}}+k_{\mathrm{s}} & -\bar{k}_{\mathrm{Is}} \cos ^{2} \alpha_{\mathrm{Is}} & 0 & 0 & 0 \\
-\bar{k}_{\mathrm{Is}} \cos ^{2} \alpha_{\mathrm{Is}} & \bar{k}_{\mathrm{Is}} \cos ^{2} \alpha_{\mathrm{Is}}+k_{\mathrm{II}} \cos ^{2} \alpha_{\mathrm{II}}+k_{\mathrm{If}} & -k_{\mathrm{II}} \cos ^{2} \alpha_{\mathrm{II}} & 0 & 0 \\
0 & -k_{\mathrm{II}} \cos ^{2} \alpha_{\mathrm{II}} & k_{\mathrm{II}} \cos ^{2} \alpha_{\mathrm{II}}+\bar{k}_{\mathrm{Io}} \cos ^{2} \alpha_{\mathrm{Io}} & -\bar{k}_{\mathrm{Io}} \cos ^{2} \alpha_{\mathrm{Io}} & 0 \\
0 & 0 & -\bar{k}_{\mathrm{Io}} \cos ^{2} \alpha_{\mathrm{Io}} & \bar{k}_{\mathrm{Io}} \cos ^{2} \alpha_{\mathrm{Io}}+k_{\mathrm{oo}} \cos ^{2} \alpha_{\mathrm{oo}} & -k_{\mathrm{oo}} \cos ^{2} \alpha_{\mathrm{oo}} \\
0 & 0 & 0 & -k_{\mathrm{oo}} \cos ^{2} \alpha_{\mathrm{oo}} & k_{\mathrm{oo}} \cos ^{2} \alpha_{\mathrm{oo}}+k_{\mathrm{o}}
\end{array}\right] \text {, }
\end{aligned}
$$

where $M_{\mathrm{s}}, M_{\mathrm{If}}, M_{\mathrm{I}}, M_{\mathrm{of}}$, and $M_{\mathrm{o}}$ are the masses of the inner stator, the inner FP, the inner rotor, the outer FP, and the outer stator, respectively; $u_{\mathrm{s}}, u_{\mathrm{If}}, u_{\mathrm{I}}, u_{\text {of }}$, and $u_{\mathrm{o}}$ are the torsional displacements of the inner stator, the inner FP, the inner rotor, the outer FP, and the outer stator, respectively; $c_{\mathrm{s}}, c_{\mathrm{If}}, c_{\mathrm{I}}, c_{\mathrm{of}}$, and $c_{\mathrm{o}}$ are the damping coefficients on the inner stator, the inner FP, the inner rotor, the outer FP, and the outer stator, respectively; $\alpha_{\text {Is }}$ is the meshing angle between the inner stator and the inner FP; $\alpha_{\mathrm{II}}$ is the meshing angle between the inner FP and the inner rotor; $\alpha_{\text {Io }}$ is the meshing angle between the inner rotor and the outer FP; $\alpha_{\mathrm{oo}}$ is the meshing angle between the outer FP and the outer stator; $k_{\mathrm{s}}, k_{\mathrm{If}}$, and $k_{\mathrm{o}}$ are the supporting stiffnesses among the inner stator, the inner $\mathrm{FP}$, the outer stator, and the foundation, respectively; $\omega_{\mathrm{e}}$ is the exciting frequency of the torque fluctuation on the outer FP; $R_{\text {of }}$ is the turning radius of the outer FP; $\Delta T_{\text {of }}$ is the torque increment on the outer FP.

Considering the time-varying periodic stiffnesses, EIMG system is a typical parametric vibration system. The dynamic differential equations of the parametric vibration system can be got by substituting (2) into (3):

$$
\mathbf{M} \ddot{\mathbf{x}}+\mathbf{c} \dot{\mathbf{x}}+\mathbf{K x}+\Delta \mathbf{K}(t) \mathbf{x}=\mathbf{F},
$$

where

$$
\left.\begin{array}{ccc}
0 & 0 & 0 \\
0 & 0 & 0 \\
k_{\mathrm{Io}} \varepsilon e^{i \omega_{\mathrm{o}} t} \cos ^{2} \alpha_{\mathrm{Io}} & -k_{\mathrm{Io}} \varepsilon e^{i \omega_{\mathrm{o}} t} \cos ^{2} \alpha_{\mathrm{Io}} & 0 \\
-k_{\mathrm{Io}} \varepsilon e^{i \omega_{\mathrm{o}} t} \cos ^{2} \alpha_{\mathrm{Io}} & k_{\mathrm{Io}} \varepsilon e^{i \omega_{0} t} \cos ^{2} \alpha_{\mathrm{Io}} & 0 \\
0 & 0 & 0
\end{array}\right],
$$

in which $\Delta \mathbf{K}(t)$ is the increment stiffness matrix.

Based on the linear system in (3), (5) can be normalized into the following form:

$$
\ddot{\mathbf{x}}_{\mathrm{N}}+\mathbf{c}_{\mathrm{N}} \dot{\mathbf{x}}_{\mathrm{N}}+\left(\mathbf{k}_{\mathrm{N}}+\Delta \mathbf{k}_{\mathrm{N}}\right) \mathbf{x}_{\mathrm{N}}=\Delta \mathbf{F}_{\mathrm{N}}
$$

In (7), $\mathbf{x}_{\mathrm{N}}, \mathbf{k}_{\mathrm{N}}, \Delta \mathbf{k}_{\mathrm{N}}(t)$, and $\Delta \mathbf{F}_{\mathrm{N}}$ are the normal displacement vector, the normal stiffness matrix, the normal increment stiffness matrix, and the normal load vector:

$$
\mathbf{x}_{\mathrm{N}}=\left[\begin{array}{lllll}
u_{\mathrm{N} 1} & u_{\mathrm{N} 2} & u_{\mathrm{N} 3} & u_{\mathrm{N} 4} & u_{\mathrm{N} 5}
\end{array}\right]^{\mathrm{T}}
$$

$$
\begin{aligned}
\Delta \mathbf{F}_{\mathrm{N}} & =\left[\begin{array}{lllll}
F_{\mathrm{N} 1} & F_{\mathrm{N} 2} & F_{\mathrm{N} 3} & F_{\mathrm{N} 4} & F_{\mathrm{N} 5}
\end{array}\right]^{\mathrm{T}} \\
\mathbf{k}_{\mathrm{N}} & =\operatorname{diag}\left(\begin{array}{lllll}
k_{\mathrm{N} 1} & k_{\mathrm{N} 2} & k_{\mathrm{N} 3} & k_{\mathrm{N} 4} & k_{\mathrm{N} 5}
\end{array}\right) \\
& =\operatorname{diag}\left(\left[\begin{array}{lllll}
\omega_{1}^{2} & \omega_{2}^{2} & \omega_{3}^{2} & \omega_{4}^{2} & \omega_{5}^{2}
\end{array}\right]\right)
\end{aligned}
$$$$
\Delta \mathbf{k}_{\mathrm{N}}(t)=\left[\begin{array}{ccc}
\cdots & \Delta k_{\mathrm{N} 1, n} & \cdots \\
\vdots & \Delta k_{\mathrm{N} k, n} & \vdots \\
\cdots & \Delta k_{\mathrm{N} 5, n} & \cdots
\end{array}\right],
$$ 


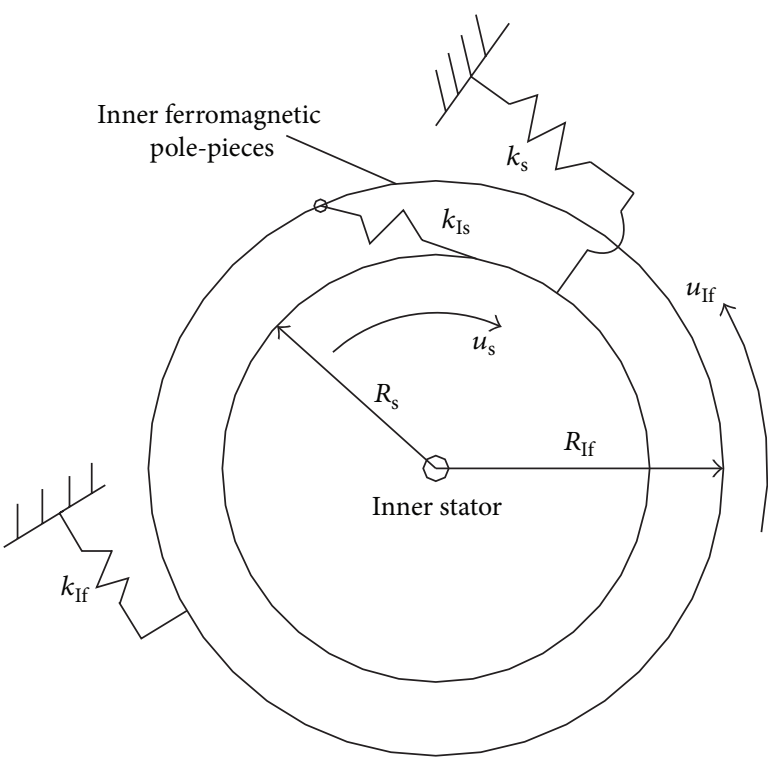

(a) The inner stator/inner FP subsystem

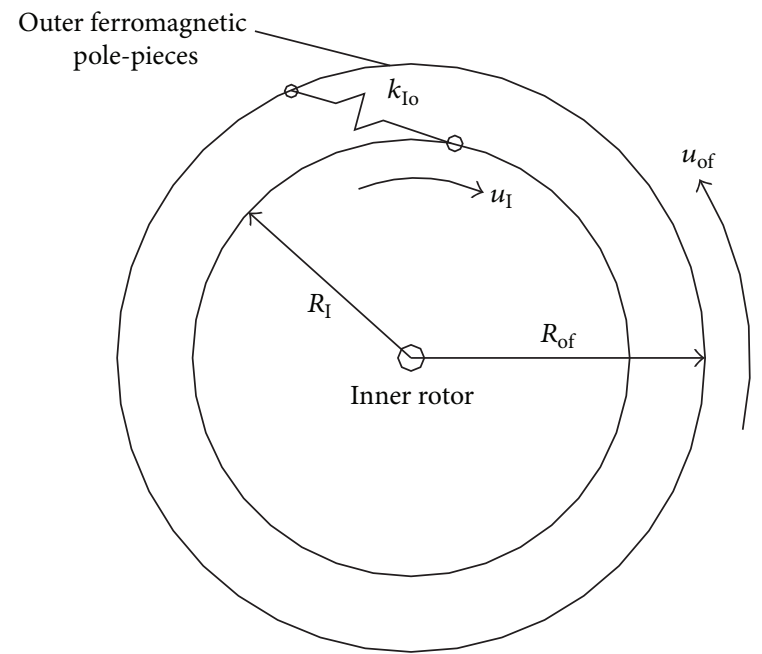

(c) The inner rotor/outer FP subsystem

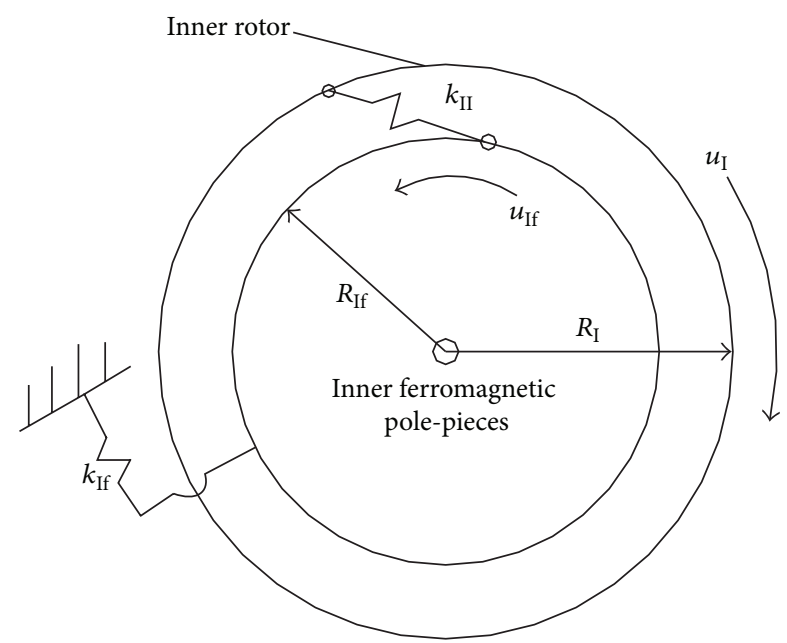

(b) The inner FP/inner rotor subsystem

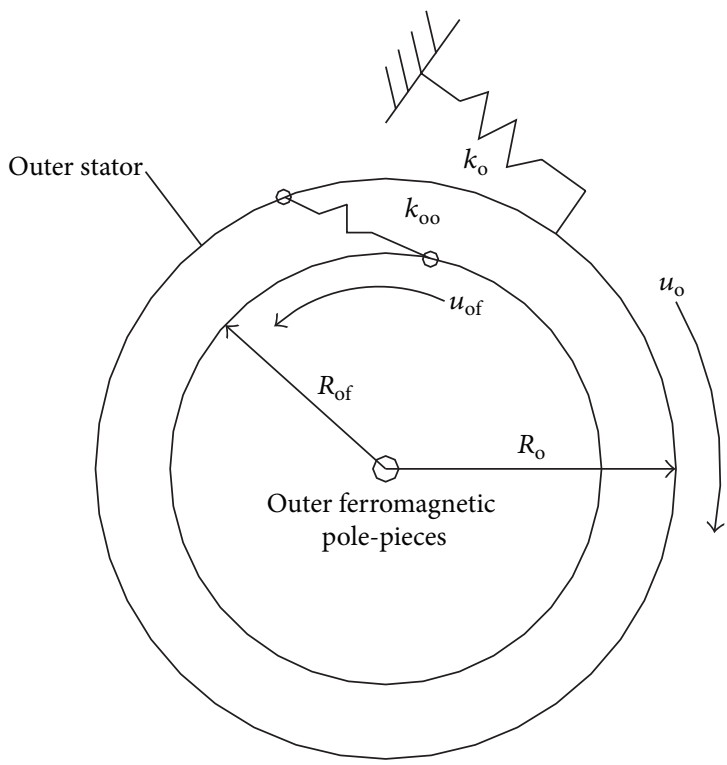

(d) The outer FP/outer rotor subsystem

Figure 4: Dynamic model of the electromechanical integrated magnetic gear.

where

$$
\begin{aligned}
\Delta k_{\mathrm{N} k, n} & =A_{k, n} e^{i \omega_{\mathrm{s}} t}+B_{k, n} e^{i \omega_{\mathrm{o}} t} ; \\
A_{k, n} & =\bar{k}_{\mathrm{Is}} \varepsilon_{\mathrm{Is}} \cos ^{2} \alpha_{\mathrm{Is}}\left(A_{\mathrm{N} 1 n}-A_{\mathrm{N} 2 n}\right)\left(A_{\mathrm{N} 1 k}-A_{\mathrm{N} 2 k}\right) ; \\
B_{k, n} & =\bar{k}_{\mathrm{Io}} \varepsilon_{\mathrm{Io}} \cos ^{2} \alpha_{\mathrm{Io}}\left(A_{\mathrm{N} 3 n}-A_{\mathrm{N} 4 n}\right)\left(A_{\mathrm{N} 3 k}-A_{\mathrm{N} 4 k}\right) ; \\
F_{\mathrm{N} k} & =\frac{-A_{\mathrm{N} 4 k} \Delta T_{\mathrm{of}} e^{i \omega_{\mathrm{e}} t}}{2 R_{\mathrm{of}}},
\end{aligned}
$$

in which $A_{\mathrm{N} k, n}, k(n)=1,2,3,4,5$, is the element on line $k$, column $n$ of the normal shape matrix $\mathbf{A}_{\mathrm{N}}$.
Because the damping matrix $\mathbf{c}$ is a diagonal matrix, the elements on the primary diagonal of the normal damping matrix $\mathbf{c}_{\mathrm{N}}$ are much bigger than other elements. So, the normal damping matrix $\mathbf{c}_{\mathrm{N}}$ in (7) can be simplified into a diagonal matrix as follows:

$$
\mathbf{c}_{\mathrm{N}}=\operatorname{diag}\left(\left[\begin{array}{lllll}
c_{\mathrm{N} 1} & c_{\mathrm{N} 2} & c_{\mathrm{N} 3} & c_{\mathrm{N} 4} & c_{\mathrm{N} 5}
\end{array}\right]\right) \text {. }
$$

\section{Resonances of EIMG System}

3.1. Main Resonance of EIMG System. The multiscale method is adopted to solve (7). Meanwhile, in order to balance the effects of the damping forces and the increment stiffnesses 
and make them into the same perturbation equation, the following assumptions are adopted:

$$
\begin{aligned}
x_{\mathrm{N} k} & =x_{\mathrm{N} k 0}\left(T_{0}, T_{1}\right)+\varepsilon x_{\mathrm{N} k 1}\left(T_{0}, T_{1}\right), \\
c_{\mathrm{N} k} & =\varepsilon c_{\mathrm{N} k}^{\prime}, \\
\Delta T_{\mathrm{of}} & =\varepsilon \Delta T_{\mathrm{of}}^{\prime},
\end{aligned}
$$

where $T_{n}=\varepsilon^{n} t$

By substituting (11) into (7) and making the same power coefficients of the small parameters $\varepsilon$ on both sides of the equations equal, the following differential equations can be obtained:

Zero power is

$$
D_{0}^{2} x_{\mathrm{N} k 0}+\omega_{k}^{2} x_{\mathrm{N} k 0}=0 .
$$

First power is

$$
\begin{aligned}
D_{0}^{2} x_{\mathrm{N} k 1}+\omega_{k}^{2} x_{\mathrm{N} k 1}= & -2 D_{0} D_{1} x_{\mathrm{N} k 0}-c_{\mathrm{N} k}^{\prime} D_{0} x_{\mathrm{N} k 0} \\
& +\sum_{n=1}^{5}\left(A_{k, n} e^{i \omega_{\mathrm{s}} t}+B_{k, n} e^{i \omega_{\mathrm{o}} t}\right) x_{\mathrm{N} k 0} \\
& -\frac{A_{\mathrm{N} 4 i} \Delta T_{\mathrm{o}}^{\prime} e^{i \omega_{\mathrm{e}} t}}{2 R_{\mathrm{of}}}+\mathrm{cc} .
\end{aligned}
$$

The general solution of (12) can be expressed:

$$
x_{\mathrm{N} k 0}=E_{k} e^{i \omega_{k} t}+\mathrm{cc},
$$

where $E_{k}$ is the initial amplitude, which can be calculated by initial energy.

By substituting (14) into (13), (13) can be rewritten as

$$
\begin{aligned}
D_{0}^{2} x_{\mathrm{N} k 1} & +\omega_{k}^{2} x_{\mathrm{N} k 1} \\
= & -2 i \omega_{k} \dot{E}_{k} e^{i \omega_{k} t}-c_{\mathrm{N} k}^{\prime} i \omega_{k} E_{k} e^{i \omega_{k} t}-\frac{A_{\mathrm{N} 4 k} \Delta T_{\mathrm{o}}^{\prime} e^{i \omega_{\mathrm{e}} t}}{2 R_{\mathrm{of}}} \\
& +\sum_{n=1}^{5}\left(A_{k, n} e^{i \omega_{\mathrm{s}} t}+B_{k, n} e^{i \omega_{\mathrm{o}} t}\right)\left(E_{k} e^{i \omega_{k} t}+\mathrm{cc}\right)+\mathrm{cc} .
\end{aligned}
$$

When the exciting frequency of the torque fluctuation on the outer FP is close to the natural frequencies of the derived EIMG system, the main resonances will occur. Here, the following assumption is introduced:

$$
\omega_{\mathrm{e}}=\omega_{3}+\varepsilon \sigma_{1} .
$$

By substituting (16) into (15) and eliminating the secular terms, the following differential equations can be obtained:

$$
\begin{array}{r}
-2 i \omega_{3} \dot{E}_{3}-c_{\mathrm{N} 3}^{\prime} i \omega_{3} E_{3}-\frac{A_{\mathrm{N} 43} \Delta T_{\mathrm{o}}^{\prime} e^{i \sigma_{1} T_{1}}}{2 R_{\mathrm{of}}}=0, \\
-2 i \omega_{m} \dot{E}_{m}-c_{\mathrm{N} m}^{\prime} i \omega_{m} E_{m}=0,
\end{array}
$$

The solution of (17) can be worked out by the method of constant variation and can be expressed as follows:

$$
\begin{aligned}
& E_{3}=G_{3} e^{-c_{\mathrm{N} 3}^{\prime} T_{1} / 2}+\frac{i A_{\mathrm{N} 43} \Delta T_{\mathrm{o}}^{\prime} e^{j \sigma_{1} T_{1}}}{2\left[R_{\mathrm{of}} \omega_{3}\left(c_{\mathrm{N} 3}^{\prime}+2 i \sigma_{1}\right)\right]}+\mathrm{cc}, \\
& E_{m}=G_{m} e^{-c_{\mathrm{N} m}^{\prime} T_{1} / 2},
\end{aligned}
$$

where $G_{m}$ is a constant.

Equation (18) can be rewritten as

$$
E_{3}=G_{3} e^{-c_{\mathrm{N} 3}^{\prime} T_{1} / 2}+\frac{A_{\mathrm{N} 43} \Delta T_{\mathrm{o}}^{\prime} e^{i\left(\theta_{1}+\sigma_{1} T_{1}\right)}}{2 \omega_{3} R_{\mathrm{of}} \sqrt{c_{\mathrm{N} 3}^{\prime 2}+4 \sigma_{1}^{2}}}+\mathrm{cc},
$$

where $\sin \theta_{1}=c_{\mathrm{N} 3}^{\prime} / \sqrt{c_{\mathrm{N} 3}^{\prime 2}+4 \sigma_{1}^{2}}, \cos \theta_{1}=2 \sigma_{1} / \sqrt{c_{\mathrm{N} 3}^{\prime 2}+4 \sigma_{1}^{2}}$.

In (19), $c_{\mathrm{Nk}}^{\prime} e^{-c_{\mathrm{Nk}}^{\prime} T_{1}}$ will be close to zero with time increasing. The stable responses of the zero order approximate analytic solution of EIMG system can be given:

$$
\begin{aligned}
x_{\mathrm{N} 30}= & \frac{A_{\mathrm{N} 43} \Delta T_{\mathrm{o}}^{\prime} e^{i\left(\theta_{1}+\sigma_{1} T_{1}\right)} e^{i \omega_{3} t}}{2 \omega_{3} R_{\mathrm{of}} \sqrt{c_{\mathrm{N} 3}^{\prime 2}+4 \sigma_{1}^{2}}}+\mathrm{cc} \\
= & \frac{A_{\mathrm{N} 43} \Delta T_{\mathrm{o}}^{\prime} \cos \left(\theta_{1}+\sigma_{1} T_{1}+\omega_{3} t\right)}{\omega_{3} R_{\mathrm{of}} \sqrt{c_{\mathrm{N} 3}^{\prime 2}+4 \sigma_{1}^{2}}},
\end{aligned}
$$

$$
x_{\mathrm{N} m 0}=0 .
$$

By substituting (19) and (20) into (15), the stable responses of the first order approximate analytic solution of EIMG system can be calculated:

$$
\begin{aligned}
x_{\mathrm{N} 31}= & \sum_{k=1}^{5}\left(\frac{E_{k} A_{3, k} e^{i\left(\omega_{\mathrm{s}} \pm \omega_{k}\right) t}}{\left(\omega_{\mathrm{s}}+\omega_{k}\right)^{2}-\omega_{3}^{2}}+\frac{E_{k} B_{3, k} e^{i\left(\omega_{\mathrm{o}} \pm \omega_{k}\right) t}}{\left(\omega_{\mathrm{o}}+\omega_{k}\right)^{2}-\omega_{3}^{2}}\right) \\
& +\mathrm{cc} \\
= & \frac{A_{\mathrm{N} 43} \Delta T_{\mathrm{o}}^{\prime} A_{33} e^{i\left[\left(\omega_{\mathrm{s}} \pm \omega_{3}\right) t+\theta_{1}+\sigma_{1} T_{1}\right]}}{2 \omega_{3} R_{\mathrm{of}}\left(\omega_{s}^{2}+2 \omega_{\mathrm{s}} \omega_{3}\right) \sqrt{c_{\mathrm{N} 3}^{\prime 2}+4 \sigma_{1}^{2}}} \\
& +\frac{A_{\mathrm{N} 43} \Delta T_{\mathrm{o}}^{\prime} B_{33} e^{i\left[\left(\omega_{\mathrm{o}} \pm \omega_{3}\right) t+\theta_{1}+\sigma_{1} T_{1}\right]}}{2 \omega_{3} R_{\mathrm{of}}\left(\omega_{\mathrm{o}}^{2}+2 \omega_{\mathrm{o}} \omega_{3}\right) \sqrt{c_{\mathrm{N} 3}^{\prime 2}+4 \sigma_{1}^{2}}}+\mathrm{cc},
\end{aligned}
$$




$$
\begin{aligned}
x_{\mathrm{N} m 1}= & \sum_{k=1}^{5}\left(\frac{E_{n} A_{3, k} e^{i\left(\omega_{\mathrm{s}} \pm \omega_{k}\right) t}}{\left(\omega_{\mathrm{s}}+\omega_{k}\right)^{2}-\omega_{k}^{2}}+\frac{E_{n} B_{3, k} e^{i\left(\omega_{\mathrm{o}} \pm \omega_{k}\right) t}}{\left(\omega_{\mathrm{o}}+\omega_{k}\right)^{2}-\omega_{k}^{2}}\right) \\
& +\frac{A_{\mathrm{N} 4, m} \Delta T_{\mathrm{o}}^{\prime} e^{i \omega_{\mathrm{e}} t}}{2 R_{\mathrm{of}}\left(\omega_{\mathrm{e}}^{2}-\omega_{m}^{2}\right)}+\mathrm{cc} \\
= & \frac{A_{\mathrm{N} 43} \Delta T_{\mathrm{o}}^{\prime} A_{33} e^{i\left[\left(\omega_{\mathrm{s}} \pm \omega_{3}\right) t+\theta_{1}+\sigma_{1} T_{1}\right]}}{2 \omega_{3} R_{\mathrm{of}}\left(\omega_{\mathrm{s}}^{2}+2 \omega_{\mathrm{s}} \omega_{3}\right) \sqrt{c_{\mathrm{N} 3}^{\prime 2}+4 \sigma_{1}^{2}}} \\
& +\frac{A_{\mathrm{N} 43} \Delta T_{\mathrm{o}}^{\prime} B_{33} e^{i\left[\left(\omega_{\mathrm{o}} \pm \omega_{3}\right) t+\theta_{1}+\sigma_{1} T_{1}\right]}}{2 \omega_{3} R_{\mathrm{of}}\left(\omega_{\mathrm{o}}^{2}+2 \omega_{\mathrm{o}} \omega_{3}\right) \sqrt{c_{\mathrm{N} 3}^{\prime 2}+4 \sigma_{1}^{2}}} \\
& +\frac{A_{\mathrm{N} 4, m} \Delta T_{\mathrm{o}}^{\prime} e^{i \omega_{\mathrm{e}} t}}{2 R_{\mathrm{of}}\left(\omega_{\mathrm{e}}^{2}-\omega_{m}^{2}\right)}+\mathrm{cc} .
\end{aligned}
$$

Equation (21) can be simplified into

$$
\begin{aligned}
x_{\mathrm{N} 31}= & \frac{A_{\mathrm{N} 43} \Delta T_{\mathrm{o}}^{\prime} A_{33} \cos \left(\omega_{\mathrm{s}} t \pm \omega_{3} t+\theta_{1}+\sigma_{1} T_{1}\right)}{2 \omega_{3} R_{\mathrm{of}}\left(\omega_{\mathrm{s}}^{2}+2 \omega_{\mathrm{s}} \omega_{3}\right) \sqrt{c_{\mathrm{N} 3}^{\prime 2}+4 \sigma_{1}^{2}}} \\
& +\frac{A_{\mathrm{N} 43} \Delta T_{\mathrm{o}}^{\prime} B_{33} \cos \left(\omega_{\mathrm{o}} t \pm \omega_{3} t+\theta_{1}+\sigma_{1} T_{1}\right)}{2 \omega_{3} R_{\mathrm{of}}\left(\omega_{\mathrm{o}}^{2}+2 \omega_{\mathrm{o}} \omega_{3}\right) \sqrt{c_{\mathrm{N} 3}^{\prime 2}+4 \sigma_{1}^{2}}} \\
& +\mathrm{cc}, \\
x_{\mathrm{N} m 1}= & \frac{A_{\mathrm{N} 43} \Delta T_{\mathrm{o}}^{\prime} A_{33} \cos \left(\omega_{\mathrm{s}} t \pm \omega_{3} t+\theta_{1}+\sigma_{1} T_{1}\right)}{2 \omega_{3} R_{\mathrm{of}}\left(\omega_{\mathrm{s}}^{2}+2 \omega_{\mathrm{s}} \omega_{3}\right) \sqrt{c_{\mathrm{N} 3}^{\prime 2}+4 \sigma_{1}^{2}}} \\
& +\frac{A_{\mathrm{N} 43} \Delta T_{\mathrm{o}}^{\prime} B_{33} \cos \left(\omega_{\mathrm{o}} t \pm \omega_{3} t+\theta_{1}+\sigma_{1} T_{1}\right)}{2 \omega_{3} R_{\mathrm{of}}\left(\omega_{\mathrm{o}}^{2}+2 \omega_{\mathrm{o}} \omega_{3}\right) \sqrt{c_{\mathrm{N} 3}^{\prime 2}+4 \sigma_{1}^{2}}} \\
& +\frac{A_{\mathrm{N} 4, m} \Delta T_{\mathrm{o}}^{\prime} \cos \omega_{\mathrm{e}} t}{2 R_{\mathrm{of}}\left(\omega_{\mathrm{e}}^{2}-\omega_{m}^{2}\right)}+\mathrm{cc} .
\end{aligned}
$$

Similarly, when the exciting frequency is close to a certain frequency $\omega_{k}$, the resonance will occur and the stable responses of EIMG system in normal coordinate system can be deduced:

$$
\begin{aligned}
x_{\mathrm{N} k 0} & =\frac{A_{\mathrm{N} 4 k} \Delta T_{\mathrm{o}}^{\prime} \cos \left(\theta_{1}+\sigma_{1} T_{1}+\omega_{k} t\right)}{\omega_{k} R_{\mathrm{of}} \sqrt{c_{\mathrm{N} k}^{\prime 2}+4 \sigma_{1}^{2}}}+\mathrm{cc}, \\
x_{\mathrm{N} m 0} & =0, \\
x_{\mathrm{N} k 1} & =\frac{A_{\mathrm{N} 4 k} \Delta T_{\mathrm{o}}^{\prime} A_{k k} \cos \left(\omega_{\mathrm{s}} t \pm \omega_{k} t+\theta_{1}+\sigma_{1} T_{1}\right)}{2 \omega_{k} R_{\mathrm{of}}\left(\omega_{\mathrm{s}}^{2}+2 \omega_{\mathrm{s}} \omega_{k}\right) \sqrt{c_{\mathrm{N} k}^{\prime 2}+4 \sigma_{1}^{2}}} \\
+ & \frac{A_{\mathrm{N} 4 k} \Delta T_{\mathrm{o}}^{\prime} B_{k k} \cos \left(\omega_{\mathrm{o}} t \pm \omega_{k} t+\theta_{1}+\sigma_{1} T_{1}\right)}{2 \omega_{k} R_{\mathrm{of}}\left(\omega_{\mathrm{o}}^{2}+2 \omega_{\mathrm{o}} \omega_{k}\right) \sqrt{c_{\mathrm{N} k}^{\prime 2}+4 \sigma_{1}^{2}}}+\mathrm{cc}
\end{aligned}
$$

$$
\begin{gathered}
x_{\mathrm{N} m 1}=\frac{A_{\mathrm{N} 4 k} \Delta T_{\mathrm{o}}^{\prime} A_{k k} \cos \left(\omega_{\mathrm{s}} t \pm \omega_{k} t+\theta_{1}+\sigma_{1} T_{1}\right)}{2 \omega_{k} R_{\mathrm{of}}\left(\omega_{\mathrm{s}}^{2}+2 \omega_{\mathrm{s}} \omega_{k}\right) \sqrt{c_{\mathrm{N} k}^{\prime 2}+4 \sigma_{1}^{2}}} \\
\cdot \frac{A_{\mathrm{N} 4 m} \Delta T_{\mathrm{o}}^{\prime} \cos \omega_{\mathrm{e}} t}{2 R_{\mathrm{of}}\left(\omega_{\mathrm{e}}^{2}-\omega_{m}^{2}\right)} \\
+\frac{A_{\mathrm{N} 4 k} \Delta T_{\mathrm{o}}^{\prime} B_{k k} \cos \left(\omega_{\mathrm{o}} t \pm \omega_{k} t+\theta_{1}+\sigma_{1} T_{1}\right)}{2 \omega_{k} R_{\mathrm{of}}\left(\omega_{\mathrm{o}}^{2}+2 \omega_{\mathrm{o}} \omega_{k}\right) \sqrt{c_{\mathrm{N} k}^{\prime 2}+4 \sigma_{1}^{2}}}+\mathrm{cc}
\end{gathered}
$$

where $m$ is any natural number except for $k, k=1,2,3,4,5$.

By substituting (23) into (11), the approximate analytical solution of EIMG system can be got by

$$
\mathbf{x}=\mathbf{A}_{\mathrm{N}}\left(\mathbf{x}_{\mathrm{N} 0}+\varepsilon \mathbf{x}_{\mathrm{N} 1}\right) \text {. }
$$

3.2. Combination Resonance of EIMG System. By the multiscale method, the following assumptions are adopted:

$$
\begin{aligned}
x_{\mathrm{N} k} & =x_{\mathrm{N} k 0}\left(T_{0}, T_{1}\right)+\varepsilon x_{\mathrm{N} k 1}\left(T_{0}, T_{1}\right), \\
\mathcal{c}_{\mathrm{N} k} & =\varepsilon c_{\mathrm{N} k}^{\prime}, \\
\omega_{\mathrm{e}} & =\omega_{3}+\omega_{\mathrm{o}}+\varepsilon \sigma_{2} .
\end{aligned}
$$

By substituting (25) into (7) and making the same power coefficients of the small parameters $\varepsilon$ on both sides of the equations equal, the following differential equations can be obtained:

Zero power is

$$
D_{0}^{2} x_{\mathrm{N} k 0}+\omega_{k}^{2} x_{\mathrm{N} k 0}=\frac{A_{\mathrm{N} 4 k} \Delta T_{\mathrm{o}} e^{i \omega_{\mathrm{e}} t}}{2 R_{\mathrm{of}}}+\mathrm{cc} .
$$

The first power is

$$
\begin{aligned}
D_{0}^{2} x_{\mathrm{N} k 1}+\omega_{k}^{2} x_{\mathrm{N} k 1}= & -2 D_{0} D_{1} x_{\mathrm{N} k 0}-c_{\mathrm{N} k}^{\prime} D_{0} x_{\mathrm{N} k 0} \\
& +\sum_{n=1}^{5}\left(A_{k, n} e^{i \omega_{\mathrm{s}} t}+B_{k, n} e^{i \omega_{\mathrm{o}} t}\right) x_{\mathrm{N} k 0} \\
+ & \mathrm{cc}, \\
& \quad k=1,2, \ldots, 5, n=1,2, \ldots, 5 .
\end{aligned}
$$

The general solution of (26) can be expressed:

$$
x_{\mathrm{N} k 0}=E_{k} e^{i \omega_{k} t}+\frac{A_{\mathrm{N} 4 k} \Delta T_{\mathrm{o}} e^{i \omega_{\mathrm{e}} t}}{2 R_{\mathrm{of}}\left(\omega_{\mathrm{e}}^{2}-\omega_{k}^{2}\right)}+\mathrm{cc} .
$$

By substituting (28) into (27) and eliminating the secular terms, the following differential equations can be obtained:

$$
\begin{aligned}
-2 i \omega_{3} \frac{\mathrm{d} E_{3}}{\mathrm{~d} T_{1}}-c_{\mathrm{N} 3}^{\prime} i \omega_{3} E_{3}+\frac{A_{\mathrm{N} 43} \Delta T_{\mathrm{o}} B_{33} e^{i \sigma_{2} T_{1}}}{2 R_{\mathrm{of}}\left(\omega_{\mathrm{e}}^{2}-\omega_{3}^{2}\right)}=0, \\
-2 i \omega_{m} \frac{\mathrm{d} E_{m}}{\mathrm{~d} T_{1}}-c_{\mathrm{N} m}^{\prime} i \omega_{m} E_{m}=0 .
\end{aligned}
$$


Shock and Vibration

9

The solution of (29) can be given:

$$
\begin{aligned}
& E_{3}=F_{3} e^{-c_{\mathrm{N} 3}^{\prime} T_{1}}+\frac{A_{\mathrm{N} 4 k} \Delta T_{\mathrm{o}} B_{33} e^{j\left(\theta_{2}+\sigma_{2} T_{1}\right)}}{2 \omega_{3} R_{\mathrm{of}}\left(\omega_{\mathrm{e}}^{2}-\omega_{3}^{2}\right) \sqrt{c_{\mathrm{N} 3}^{\prime 2}+4 \sigma_{2}^{2}}}+\mathrm{cc}, \\
& E_{m}=F_{m} e^{-c_{\mathrm{N} m}^{\prime} T_{1}},
\end{aligned}
$$

where $\sin \theta_{2}=c_{\mathrm{N} 3}^{\prime} / \sqrt{c_{\mathrm{N} 3}^{\prime 2}+4 \sigma_{2}^{2}}, \cos \theta_{2}=2 \sigma_{2} / \sqrt{c_{\mathrm{N} 3}^{\prime 2}+4 \sigma_{2}^{2}}$.

In (30), $F_{k} e^{-c_{\mathrm{N} k}^{\prime} T_{1}}$ will gradually be close to zero with time going on. The stable responses of the zero order approximate analytic solutions of EIMG system can be given:

$$
\begin{aligned}
& x_{\mathrm{N} 30} \\
& =\frac{A_{\mathrm{N} 43} \Delta T_{\mathrm{o}}}{2 R_{\mathrm{of}}\left(\omega_{\mathrm{e}}^{2}-\omega_{3}^{2}\right)}\left[\frac{B_{33} e^{i\left(\omega_{3} t+\theta_{2}+\sigma T_{1}\right)}}{\omega_{3} \sqrt{c_{\mathrm{N} 3}^{\prime 2}+4 \sigma_{2}^{2}}}+e^{i \omega_{\mathrm{e}} t}+\mathrm{cc}\right], \\
& x_{\mathrm{N} m 0}=\frac{A_{\mathrm{N} 4 m} \Delta T_{\mathrm{o}} e^{i \omega_{\mathrm{e}} t}}{2 R_{\mathrm{of}}\left(\omega_{\mathrm{e}}^{2}-\omega_{m}^{2}\right)}+\mathrm{cc} .
\end{aligned}
$$

By substituting (28) and (31) into (27), the stable responses of the first order approximate analytic solution of (27) can be calculated:

$$
\begin{aligned}
& u_{\mathrm{N} 31}=\sum_{n=1}^{5}\left(\frac{A_{3 n} E_{n} e^{i\left(\omega_{\mathrm{s}} \pm \omega_{j}\right) t}}{\left(\omega_{\mathrm{s}} \pm \omega_{n}\right)^{2}-\omega_{3}^{2}}+\frac{B_{3 n} E_{n} e^{i\left(\omega_{\mathrm{o}} \pm \omega_{j}\right) t}}{\left(\omega_{\mathrm{o}} \pm \omega_{n}\right)^{2}-\omega_{3}^{2}}\right. \\
& \left.+\frac{A_{43} \Delta T_{\mathrm{o}} A_{3 n} e^{i\left(\omega_{\mathrm{s}} \pm \omega_{\mathrm{e}}\right) t}}{2 R_{\mathrm{of}}\left(\omega_{\mathrm{e}}^{2}-\omega_{n}^{2}\right)\left[\left(\omega_{\mathrm{s}} \pm \omega_{\mathrm{e}}\right)^{2}-\omega_{3}^{2}\right]}\right) \\
& +\sum_{m=1,2,4,5} \frac{A_{43} \Delta T_{\mathrm{o}} B_{3 m} e^{i\left(\omega_{\mathrm{o}} \pm \omega_{\mathrm{e}}\right) t}}{2 R_{\mathrm{of}}\left(\omega_{\mathrm{e}}^{2}-\omega_{m}^{2}\right)\left[\left(\omega_{\mathrm{o}} \pm \omega_{\mathrm{e}}\right)^{2}-\omega_{3}^{2}\right]}+\mathrm{cc} \\
& =\frac{A_{\mathrm{N} 43} \Delta T_{\mathrm{o}}}{2 R_{\mathrm{of}}\left(\omega_{\mathrm{e}}^{2}-\omega_{3}^{2}\right)}\left[\frac{A_{33} B_{33} e^{i\left[\left(\omega_{\mathrm{s}} \pm \omega_{3}\right) t+\theta_{2}+\varepsilon \sigma_{2} t+\omega_{3} t\right]}}{\omega_{3} \sqrt{c_{\mathrm{N} 3}^{\prime 2}+4 \sigma_{2}^{2}}\left[\left(\omega_{\mathrm{s}} \pm \omega_{3}\right)^{2}-\omega_{3}^{2}\right]}\right. \\
& \left.+\frac{B_{33}^{2} e^{i\left[\left(\omega_{0} \pm \omega_{3}\right) t+\theta_{2}+\varepsilon \sigma_{2} t+\omega_{3} t\right]}}{\omega_{3} \sqrt{c_{\mathrm{N} 3}^{\prime 2}+4 \sigma_{2}^{2}}\left[\left(\omega_{\mathrm{o}} \pm \omega_{3}\right)^{2}-\omega_{3}^{2}\right]}\right] \\
& +\sum_{n=1}^{5} \frac{A_{\mathrm{N} 43} \Delta T_{\mathrm{o}} A_{3 n} e^{i\left(\omega_{\mathrm{s}} \pm \omega_{\mathrm{e}}\right) t}}{2 R_{\mathrm{of}}\left(\omega_{\mathrm{e}}^{2}-\omega_{n}^{2}\right)\left[\left(\omega_{\mathrm{s}} \pm \omega_{\mathrm{e}}\right)^{2}-\omega_{3}^{2}\right]} \\
& +\sum_{m=1,2,4,5} \frac{A_{\mathrm{N} 43} \Delta T_{\mathrm{o}} B_{3 m} e^{i\left(\omega_{\mathrm{o}} \pm \omega_{j}\right) t}}{2 R_{\mathrm{of}}\left(\omega_{\mathrm{e}}^{2}-\omega_{m}^{2}\right)\left[\left(\omega_{\mathrm{o}} \pm \omega_{m}\right)^{2}-\omega_{3}^{2}\right]}+\mathrm{cc}
\end{aligned}
$$

$$
\begin{aligned}
& u_{\mathrm{N} m 1}=\sum_{n=1}^{5}\left(\frac{A_{3 n} E_{n} e^{i\left(\omega_{\mathrm{s}} \pm \omega_{\mathrm{j}}\right) t}}{\left(\omega_{\mathrm{s}} \pm \omega_{n}\right)^{2}-\omega_{3}^{2}}+\frac{B_{3 n} E_{n} e^{i\left(\omega_{\mathrm{o}} \pm \omega_{\mathrm{j}}\right) t}}{\left(\omega_{\mathrm{o}} \pm \omega_{n}\right)^{2}-\omega_{3}^{2}}\right) \\
& +\sum_{n=1}^{5} \frac{A_{\mathrm{N} 43} \Delta T_{\mathrm{o}}}{2 R_{\mathrm{of}}\left(\omega_{\mathrm{e}}^{2}-\omega_{n}^{2}\right)}\left(\frac{A_{3 n} e^{i\left(\omega_{\mathrm{s}} \pm \omega_{\mathrm{e}}\right) t}}{\left(\omega_{\mathrm{s}} \pm \omega_{\mathrm{e}}\right)^{2}-\omega_{3}^{2}}\right. \\
& \left.+\frac{B_{3 n} e^{i\left(\omega_{\mathrm{o}} \pm \omega_{\mathrm{e}}\right) t}}{\left(\omega_{\mathrm{o}} \pm \omega_{\mathrm{e}}\right)^{2}-\omega_{3}^{2}}\right)+c c \\
& =\frac{A_{\mathrm{N} 43} \Delta T_{\mathrm{o}}}{2 R_{\mathrm{of}}\left(\omega_{\mathrm{e}}^{2}-\omega_{3}^{2}\right)}\left[\frac{A_{33} B_{33} e^{i\left[\left(\omega_{\mathrm{s}} \pm \omega_{3}\right) t+\theta_{2}+\varepsilon \sigma_{2} t+\omega_{3} t\right]}}{\omega_{3} \sqrt{c_{\mathrm{N} 3}^{\prime 2}+4 \sigma_{2}^{2}}\left[\left(\omega_{\mathrm{s}} \pm \omega_{3}\right)^{2}-\omega_{3}^{2}\right]}\right. \\
& \left.+\frac{B_{33}^{2} e^{i\left[\left(\omega_{0} \pm \omega_{3}\right) t+\theta_{2}+\varepsilon \sigma_{2} t+\omega_{3} t\right]}}{\omega_{3} \sqrt{c_{\mathrm{N} 3}^{\prime 2}+4 \sigma_{2}^{2}}\left[\left(\omega_{\mathrm{o}} \pm \omega_{3}\right)^{2}-\omega_{3}^{2}\right]}\right] \\
& +\sum_{n=1}^{5} \frac{A_{\mathrm{N} 43} \Delta T_{\mathrm{o}}}{2 R_{\mathrm{of}}\left(\omega_{\mathrm{e}}^{2}-\omega_{n}^{2}\right)}\left(\frac{A_{3 n} e^{i\left(\omega_{\mathrm{s}} \pm \omega_{\mathrm{e}}\right) t}}{\left(\omega_{\mathrm{s}} \pm \omega_{\mathrm{e}}\right)^{2}-\omega_{3}^{2}}\right. \\
& \left.+\frac{B_{3 n} e^{i\left(\omega_{\mathrm{o}} \pm \omega_{\mathrm{e}}\right) t}}{\left(\omega_{\mathrm{o}} \pm \omega_{\mathrm{e}}\right)^{2}-\omega_{3}^{2}}\right)+\mathrm{cc} .
\end{aligned}
$$

Equation (32) can be simplified into

$u_{\mathrm{N} 31}$

$$
\begin{aligned}
& =\frac{A_{\mathrm{N} 43} \Delta T_{\mathrm{o}}}{2 R_{\mathrm{of}}\left(\omega_{\mathrm{e}}^{2}-\omega_{3}^{2}\right)}\left[\frac{A_{33} B_{33} \cos \left[\left(\omega_{\mathrm{s}} \pm \omega_{3}\right) t+\theta_{2}+\varepsilon \sigma_{2} t+\omega_{3} t\right]}{\omega_{3} \sqrt{c_{\mathrm{N} 3}^{\prime 2}+4 \sigma_{2}^{2}}\left[\left(\omega_{\mathrm{s}} \pm \omega_{3}\right)^{2}-\omega_{3}^{2}\right]}\right. \\
& \left.+\frac{B_{33}^{2} \cos \left[\left(\omega_{\mathrm{o}} \pm \omega_{3}\right) t+\theta_{2}+\varepsilon \sigma_{2} t+\omega_{3} t\right]}{\omega_{3} \sqrt{c_{\mathrm{N} 3}^{\prime 2}+4 \sigma_{2}^{2}}\left[\left(\omega_{\mathrm{o}} \pm \omega_{3}\right)^{2}-\omega_{3}^{2}\right]}\right] \\
& +\sum_{n=1}^{5} \frac{A_{\mathrm{N} 43} \Delta T_{\mathrm{o}} A_{3 n} \cos \left(\omega_{\mathrm{s}} \pm \omega_{\mathrm{j}}\right) t}{2 R_{\mathrm{of}}\left(\omega_{\mathrm{e}}^{2}-\omega_{n}^{2}\right)\left[\left(\omega_{\mathrm{s}} \pm \omega_{n}\right)^{2}-\omega_{3}^{2}\right]} \\
& +\sum_{m=1,2,4,5} \frac{A_{\mathrm{N} 43} \Delta T_{\mathrm{o}} B_{3 m} \cos \left(\omega_{\mathrm{o}} \pm \omega_{m}\right) t}{\left.\mathrm{e}-\omega_{m}^{2}\right)\left[\left(\omega_{\mathrm{o}} \pm \omega_{m}\right)^{2}-\omega_{3}^{2}\right]}+\mathrm{cc},
\end{aligned}
$$

$u_{\mathrm{N} m 1}$

$$
\begin{aligned}
& =\frac{A_{\mathrm{N} 43} \Delta T_{\mathrm{o}}}{2 R_{\mathrm{of}}\left(\omega_{\mathrm{e}}^{2}-\omega_{3}^{2}\right)}\left[\frac{A_{33} B_{33} \cos \left[\left(\omega_{\mathrm{s}} \pm \omega_{3}\right) t+\theta_{2}+\varepsilon \sigma_{2} t+\omega_{3} t\right]}{\omega_{3} \sqrt{c_{\mathrm{N} 3}^{\prime 2}+4 \sigma_{2}^{2}}\left[\left(\omega_{\mathrm{s}} \pm \omega_{3}\right)^{2}-\omega_{3}^{2}\right]}\right. \\
& \left.+\frac{B_{33}^{2} \cos \left[\left(\omega_{\mathrm{o}} \pm \omega_{3}\right) t+\theta_{2}+\varepsilon \sigma_{2} t+\omega_{3} t\right]}{\omega_{3} \sqrt{c_{\mathrm{N} 3}^{\prime 2}+4 \sigma_{2}^{2}}\left[\left(\omega_{\mathrm{o}} \pm \omega_{3}\right)^{2}-\omega_{3}^{2}\right]}\right] \\
& +\sum_{n=1}^{5} \frac{A_{\mathrm{N} 43} \Delta T_{\mathrm{o}}}{2 R_{\mathrm{of}}\left(\omega_{\mathrm{e}}^{2}-\omega_{n}^{2}\right)}\left(\frac{A_{3 n} \cos \left(\omega_{\mathrm{s}} \pm \omega_{n}\right) t}{\left(\omega_{\mathrm{s}} \pm \omega_{n}\right)^{2}-\omega_{3}^{2}}\right. \\
& \left.+\frac{B_{3 n} \cos \left(\omega_{\mathrm{o}} \pm \omega_{n}\right) t}{\left(\omega_{\mathrm{o}} \pm \omega_{n}\right)^{2}-\omega_{3}^{2}}\right)+\mathrm{cc} .
\end{aligned}
$$


Similarly, when the exciting frequency is close to a certain frequency $\omega_{k}, k=1,2,3,4,5$, the stable responses of EIMG system in normal coordinate system can be deduced:

$$
\begin{aligned}
& x_{\mathrm{N} k 0}=\frac{A_{\mathrm{N} 4 k} \Delta T_{\mathrm{o}}}{2 R_{\mathrm{of}}\left(\omega_{\mathrm{e}}^{2}-\omega_{k}^{2}\right)}\left[\frac{B_{k k} e^{i\left(\omega_{k} t+\theta_{2}+\sigma T_{1}\right)}}{\omega_{k} \sqrt{c_{\mathrm{Nk}}^{\prime 2}+4 \sigma_{2}^{2}}}+e^{i \omega_{\mathrm{e}} t}\right]+\mathrm{cc}, \\
& x_{\mathrm{N} m 0}=\frac{A_{\mathrm{N} 4 m} \Delta T_{\mathrm{o}} e^{i \omega_{\mathrm{e}} t}}{2 R_{\mathrm{of}}\left(\omega_{\mathrm{e}}^{2}-\omega_{m}^{2}\right)}+\mathrm{cc}, \\
& u_{\mathrm{N} k 1} \\
& =\frac{A_{\mathrm{N} 4 k} \Delta T_{\mathrm{o}}}{2 R_{\mathrm{of}}\left(\omega_{\mathrm{e}}^{2}-\omega_{k}^{2}\right)}\left[\frac{A_{k k} B_{k k} \cos \left[\left(\omega_{\mathrm{s}} \pm \omega_{k}\right) t+\theta_{2}+\varepsilon \sigma_{2} t+\omega_{k} t\right]}{\omega_{k} \sqrt{c_{\mathrm{N} k}^{\prime 2}+4 \sigma_{2}^{2}}\left[\left(\omega_{\mathrm{s}} \pm \omega_{k}\right)^{2}-\omega_{k}^{2}\right]}\right. \\
& \left.+\frac{B_{k k}^{2} \cos \left[\left(\omega_{\mathrm{o}} \pm \omega_{k}\right) t+\theta_{2}+\varepsilon \sigma_{2} t+\omega_{k} t\right]}{\omega_{k} \sqrt{c_{\mathrm{Nk}}^{\prime 2}+4 \sigma_{2}^{2}}\left[\left(\omega_{\mathrm{o}} \pm \omega_{k}\right)^{2}-\omega_{k}^{2}\right]}\right] \\
& +\sum_{n=1}^{5} \frac{A_{4 k} \Delta T_{\mathrm{o}} A_{k k} \cos \left(\omega_{\mathrm{s}} \pm \omega_{n}\right) t}{2 R_{\mathrm{of}}\left(\omega_{\mathrm{e}}^{2}-\omega_{n}^{2}\right)\left[\left(\omega_{\mathrm{s}} \pm \omega_{n}\right)^{2}-\omega_{k}^{2}\right]} \\
& +\sum_{m \neq k} \frac{A_{4 k} \Delta T_{\mathrm{o}} B_{m m} \cos \left(\omega_{\mathrm{o}} \pm \omega_{m}\right) t}{2 R_{\mathrm{o}}\left(\omega_{\mathrm{e}}^{2}-\omega_{m}^{2}\right)\left[\left(\omega_{\mathrm{o}} \pm \omega_{m}\right)^{2}-\omega_{k}^{2}\right]}+\mathrm{cc} \\
& u_{\mathrm{N} m 1} \\
& =\frac{A_{\mathrm{N} 4 k} \Delta T_{\mathrm{o}}}{2 R_{\mathrm{of}}\left(\omega_{\mathrm{e}}^{2}-\omega_{k}^{2}\right)}\left[\frac{A_{m m} B_{k k} \cos \left[\left(\omega_{\mathrm{s}} \pm \omega_{k}\right) t+\theta_{2}+\varepsilon \sigma_{2} t+\omega_{k} t\right]}{\omega_{m} \sqrt{c_{\mathrm{N} m}^{\prime 2}+4 \sigma_{2}^{2}}\left[\left(\omega_{\mathrm{s}} \pm \omega_{n}\right)^{2}-\omega_{k}^{2}\right]}\right. \\
& \left.+\frac{B_{m m} B_{k k} \cos \left[\left(\omega_{\mathrm{o}} \pm \omega_{k}\right) t+\theta_{2}+\varepsilon \sigma_{2} t+\omega_{k} t\right]}{\omega_{m} \sqrt{c_{\mathrm{N} m}^{\prime 2}+4 \sigma_{2}^{2}}\left[\left(\omega_{\mathrm{o}} \pm \omega_{m}\right)^{2}-\omega_{k}^{2}\right]}\right] \\
& +\sum_{n=1}^{5} \frac{A_{4 n} \Delta T_{\mathrm{o}}}{2 R_{\mathrm{of}}\left(\omega_{\mathrm{e}}^{2}-\omega_{n}^{2}\right)}\left(\frac{A_{3 n} \cos \left(\omega_{\mathrm{s}} \pm \omega_{n}\right) t}{\left(\omega_{\mathrm{s}} \pm \omega_{n}\right)^{2}-\omega_{k}^{2}}\right. \\
& \left.+\frac{B_{3 n} \cos \left(\omega_{\mathrm{o}} \pm \omega_{m}\right) t}{\left(\omega_{\mathrm{o}} \pm \omega_{n}\right)^{2}-\omega_{k}^{2}}\right)+\mathrm{cc}
\end{aligned}
$$

where $m$ is any natural number except for $k$.

Meanwhile, the combination resonance responses can be worked out when the exciting frequency is close to $\omega_{k}+\omega_{s}$ :

$$
\begin{aligned}
x_{\mathrm{N} k 0}= & \frac{A_{\mathrm{N} 4 k} \Delta T_{\mathrm{o}}}{2 R_{\mathrm{of}}\left(\omega_{\mathrm{e}}^{2}-\omega_{k}^{2}\right)}\left[\frac{A_{k k} e^{i\left(\omega_{k} t+\theta_{3}+\sigma T_{1}\right)}}{\omega_{k} \sqrt{c_{\mathrm{N} k}^{\prime 2}+4 \sigma_{3}^{2}}}+e^{i \omega_{\mathrm{e}} t}\right] \\
& +\mathrm{cc}, \\
x_{\mathrm{N} m 0}= & \frac{A_{\mathrm{N} 4 n} \Delta T_{\mathrm{o}} e^{i \omega_{\mathrm{e}} t}}{2 R_{\mathrm{of}}\left(\omega_{\mathrm{e}}^{2}-\omega_{n}^{2}\right)}+\mathrm{cc},
\end{aligned}
$$

TABLE 2: Characteristic parameters of the example EIMG system.

\begin{tabular}{lcccc}
\hline$k_{\mathrm{oo}}(\mathrm{N} / \mathrm{m})$ & $k_{\mathrm{Io}}(\mathrm{N} / \mathrm{m})$ & $k_{\mathrm{II}}(\mathrm{N} / \mathrm{m})$ & $\alpha_{\mathrm{Io}}\left({ }^{\circ}\right)$ & $\alpha_{\mathrm{oo}}\left(^{\circ}\right)$ \\
$8.6444 \times 10^{5}$ & $3.1446 \times 10^{5}$ & $4.5467 \times 10^{5}$ & 0.1818 & 0.1014 \\
\hline$k_{\mathrm{Is}}(\mathrm{N} / \mathrm{m})$ & $k_{\mathrm{If}}(\mathrm{N} / \mathrm{m})$ & $k_{\mathrm{o}}(\mathrm{N} / \mathrm{m})$ & $\alpha_{\mathrm{II}}\left({ }^{\circ}\right)$ & $\alpha_{\mathrm{Is}}\left(^{\circ}\right)$ \\
$2.0416 \times 10^{5}$ & $3 \times 10^{6}$ & $3 \times 10^{6}$ & 0.1435 & 0.2022 \\
\hline$c_{\mathrm{s}}(\mathrm{N} /(\mathrm{m} / \mathrm{s}))$ & $c_{\mathrm{If}}(\mathrm{N} /(\mathrm{m} / \mathrm{s}))$ & $c_{\mathrm{of}}(\mathrm{N} /(\mathrm{m} / \mathrm{s}))$ & $M_{\mathrm{s}}(\mathrm{kg})$ & $M_{\mathrm{If}}(\mathrm{kg})$ \\
0.1 & 0.1 & 0.05 & 1.3 & 1.3 \\
\hline$c_{\mathrm{I}}(\mathrm{N} /(\mathrm{m} / \mathrm{s}))$ & $c_{\mathrm{o}}(\mathrm{N} /(\mathrm{m} / \mathrm{s}))$ & $\Delta T_{\mathrm{of}}(\mathrm{N} \cdot \mathrm{m})$ & $M_{\mathrm{of}}(\mathrm{kg})$ & $M_{\mathrm{o}}(\mathrm{kg})$ \\
0.05 & 0.1 & 5 & 6.2 & 7.7 \\
\hline$M_{\mathrm{I}}(\mathrm{kg})$ & $R_{\mathrm{o}}$ & & & \\
11 & 219 & & &
\end{tabular}

where $\omega_{\mathrm{e}}=\omega_{k}+\omega_{\mathrm{s}}+\varepsilon \sigma_{3}, \sin \theta_{3}=c_{\mathrm{N} k}^{\prime} / \sqrt{c_{\mathrm{N} k}^{\prime 2}+4 \sigma_{3}^{2}}, \cos \theta_{3}=$ $2 \sigma_{3} / \sqrt{c_{\mathrm{N} k}^{\prime 2}+4 \sigma_{3}^{2}}$. Consider

$$
\begin{aligned}
& u_{\mathrm{N} k 1} \\
& =\frac{A_{\mathrm{N} 4 k} \Delta T_{\mathrm{o}}}{2 R_{\mathrm{of}}\left(\omega_{\mathrm{e}}^{2}-\omega_{k}^{2}\right)}\left[\frac{A_{k k}^{2} \cos \left[\left(\omega_{\mathrm{s}} \pm \omega_{k}\right) t+\theta_{3}+\varepsilon \sigma_{2} t+\omega_{k} t\right]}{\omega_{k} \sqrt{\mathcal{c}_{\mathrm{N} k}^{\prime 2}+4 \sigma_{2}^{2}}\left[\left(\omega_{\mathrm{s}} \pm \omega_{k}\right)^{2}-\omega_{k}^{2}\right]}\right. \\
& \left.+\frac{A_{k k} B_{k k} \cos \left[\left(\omega_{\mathrm{o}} \pm \omega_{k}\right) t+\theta_{3}+\varepsilon \sigma_{2} t+\omega_{k} t\right]}{\omega_{k} \sqrt{c_{\mathrm{N} k}^{\prime 2}+4 \sigma_{2}^{2}}\left[\left(\omega_{\mathrm{o}} \pm \omega_{k}\right)^{2}-\omega_{k}^{2}\right]}\right] \\
& +\sum_{m \neq k} \frac{A_{4 k} \Delta T_{\mathrm{o}} A_{m m} \cos \left(\omega_{\mathrm{s}} \pm \omega_{m}\right) t}{2 R_{\mathrm{of}}\left(\omega_{\mathrm{e}}^{2}-\omega_{m}^{2}\right)\left[\left(\omega_{\mathrm{s}} \pm \omega_{m}\right)^{2}-\omega_{k}^{2}\right]} \\
& +\sum_{n=1}^{5} \frac{A_{4 k} \Delta T_{\mathrm{o}} B_{k k} \cos \left(\omega_{\mathrm{o}} \pm \omega_{n}\right) t}{2 R_{\mathrm{o}}\left(\omega_{\mathrm{e}}^{2}-\omega_{n}^{2}\right)\left[\left(\omega_{\mathrm{o}} \pm \omega_{n}\right)^{2}-\omega_{k}^{2}\right]}+\mathrm{cc}, \\
& u_{\mathrm{N} m 1} \\
& =\frac{A_{\mathrm{N} 4 k} \Delta T_{\mathrm{o}}}{2 R_{\mathrm{of}}\left(\omega_{\mathrm{e}}^{2}-\omega_{k}^{2}\right)}\left[\frac{A_{k k}^{2} \cos \left[\left(\omega_{\mathrm{s}} \pm \omega_{k}\right) t+\theta_{3}+\varepsilon \sigma_{2} t+\omega_{k} t\right]}{\omega_{m} \sqrt{c_{\mathrm{N} m}^{\prime 2}+4 \sigma_{2}^{2}}\left[\left(\omega_{\mathrm{s}} \pm \omega_{m}\right)^{2}-\omega_{k}^{2}\right]}\right. \\
& \left.+\frac{A_{k k} B_{k k} \cos \left[\left(\omega_{\mathrm{o}} \pm \omega_{k}\right) t+\theta_{3}+\varepsilon \sigma_{2} t+\omega_{k} t\right]}{\omega_{m} \sqrt{c_{\mathrm{N} m}^{\prime 2}+4 \sigma_{2}^{2}}\left[\left(\omega_{\mathrm{o}} \pm \omega_{m}\right)^{2}-\omega_{k}^{2}\right]}\right] \\
& +\sum_{n=1}^{5} \frac{A_{4 n} \Delta T_{\mathrm{o}}}{2 R_{\mathrm{of}}\left(\omega_{\mathrm{e}}^{2}-\omega_{n}^{2}\right)}\left(\frac{A_{3 n} \cos \left(\omega_{\mathrm{s}} \pm \omega_{n}\right) t}{\left(\omega_{\mathrm{s}} \pm \omega_{n}\right)^{2}-\omega_{k}^{2}}\right. \\
& \left.+\frac{B_{3 n} \cos \left(\omega_{\mathrm{o}} \pm \omega_{n}\right) t}{\left(\omega_{\mathrm{o}} \pm \omega_{n}\right)^{2}-\omega_{k}^{2}}\right)+\mathrm{cc}
\end{aligned}
$$

\section{Results and Discussion}

4.1. Modal Characteristics of EIMG System. The dynamic parameters of the example EIMG system shown in Table 1 can be given in Table 2. By substituting the dynamic parameters into (3) and neglecting the external load, the modal frequencies and corresponding modal shapes can be calculated, which are shown in Table 3 .

Table 3 illustrates that there are five different modes, in which the natural frequencies and the modal shapes are 
TABLE 3: Modal frequencies and modal shapes of EIMG system.

\begin{tabular}{|c|c|c|c|c|c|}
\hline Mode type & IRRM & OFRM & OSRM & ISRM & IFRM \\
\hline Natural frequencies $(\mathrm{rad} / \mathrm{s})$ & 277.8 & 456.4 & 827.7 & 1322.5 & 1624.3 \\
\hline \multirow{5}{*}{ Mode shapes } & 0.0489 & -0.0333 & 0.0090 & 1.0000 & 0.3952 \\
\hline & 0.2860 & -0.1806 & 0.0348 & 0.3804 & -1.0000 \\
\hline & 1.0000 & -0.5953 & 0.0892 & -0.0195 & 0.0330 \\
\hline & 0.6747 & 1.0000 & -0.9161 & 0.0014 & -0.0014 \\
\hline & 0.3705 & 0.8125 & 1.0000 & -0.0003 & 0.0001 \\
\hline
\end{tabular}

completely different. When the natural frequencies increase, the relative displacements of the inner rotor, the outer FP, the outer stator, the inner stator, and the inner FP come to the maximum in sequence. So, the corresponding modal shapes of the natural frequencies from low to high are named the rotational modes of the inner rotor, the outer FP, the outer stator, the inner stator, and the inner FP (IRRM, OFRM, ORSM, IRSM, and IFRM), respectively. For example, when the natural frequency is $456.4 \mathrm{rad} / \mathrm{s}$, the relative displacement of the outer FP is maximal and the corresponding mode shape is named rotational mode of the outer FP.

The inner stator, the inner FP, and the outer stator are fixed on the frame of EIMG system. The inner rotor and the outer FP are coupled with other components by the electromagnetic coupling forces. Because the electromagnetic coupling stiffnesses are much smaller than the mechanical support stiffnesses, the modal frequencies of ROSM, RISM, and RIFM are bigger than the modal frequencies of RIRM and ROFM.

4.2. Main Resonances of EIMG System. By substituting the parameters of the example EIMG system into (24), the main resonances can be drawn and shown in Figure 5.

The resonance amplitudes vary widely in the main resonance of the EIMG system. When $\omega_{\mathrm{e}}$ is close to $\omega_{1}$, except the inner rotor, the resonance displacements of the inner and the outer FP are bigger than others. When $\omega_{\mathrm{e}}$ is close to $\omega_{2}$, except the outer FP, the resonance displacements of the inner rotor and the outer stator are bigger than others. When $\omega_{\mathrm{e}}$ is close to $\omega_{3}$, the resonance displacements of the outer FP and the outer stator are much bigger than others. When $\omega_{\mathrm{e}}$ is close to $\omega_{4}$ or $\omega_{5}$, the resonance displacements of the inner stator and the inner FP are much bigger than others.

When the exciting frequency is close to the rotational modal frequencies of the inner rotor, the outer FP, and the outer stator, the resonance amplitudes are much bigger than other resonances of EIMG system.

4.3. Combination Resonances of EIMG System. By substituting the parameters of the example EIMG system into (34) to (36), the time and frequency responses of the combination resonances can be drawn and shown in Figures 6 and 7 .

Figure 6 shows that the dynamic displacements of the inner rotor, the outer FP, the outer stator, the inner stator, and the inner FP will reach maximum, when the exciting frequency increases from small to big and the combination resonances occur. The dominant frequency of the combination resonances is the natural frequency $\omega_{k}$ of EIMG system rather than the exciting frequency $\omega_{\mathrm{e}}$.

When the combination occurs, the mode energies of IRRM, OFRM, ORSM, IRSM, and IFRM will be motivated, respectively. The amplitudes of inner rotor, the outer FP, the outer stator, the inner stator, and the inner FP will reach maximum, respectively. Considering the electromagnetic coupling interaction, the amplitudes of components will have a big difference, because the adjacent components are affected greatly and the separated components are affected less. For example, when $\omega_{\mathrm{e}} \approx \omega_{1}+\omega_{\mathrm{o}}$, the mode energy of IRRM will be motivated and the amplitude of the inner rotor will be maximal. Meanwhile, the amplitudes of the inner and outer FP are bigger and the resonances of the inner and outer stator are relatively smaller. Compared with the main resonances, the amplitudes in combination resonances are much smaller.

When the exciting frequency is close to the combination frequency between a certain frequency and the meshing frequency $\omega_{\mathrm{s}}$, namely, $\omega_{\mathrm{e}} \approx \omega_{k}+\omega_{\mathrm{s}}$, the combination resonances have similar characteristics with $\omega_{\mathrm{e}} \approx \omega_{k}+\omega_{\mathrm{o}}$. Meanwhile, the resonance amplitudes are smaller. Because the inner stator is not adjacent with the outer FP and the outer exciting resource is on the outer FP, the influence of the meshing stiffness wave $\Delta k_{\mathrm{Is}}$ on the outer FP is very small. So the combination resonance will occur when the exciting frequency $\omega_{\mathrm{e}}$ must be very close to the combination frequency $\omega_{k}+\omega_{s}$ and the resonance amplitude is very small.

4.4. Discussion on Resonances of EIMG System. All the components in the EIMG system are coupled by the electromagnetic fields and the electromagnetic coupling stiffnesses are much smaller than the supporting stiffnesses of the components. Because the interactions among all the components are soft-contact, the adjacent components are affected greatly and the separated components are affected less. So, the relative amplitudes of components in mode shapes have great difference. Meanwhile, the relative amplitudes of components in main resonances and the combination resonances have large difference too.

Considering the mode shape characteristic of EIMG system, the equivalent loads on the inner rotor, the outer FP, and the outer stator are much bigger than the equivalent loads on the inner stator and the inner FP. Meanwhile, because the outer FP is the output component, the mode energies of OFRM, IRRM, and OFRM are motivated easily and the corresponding main resonances are relatively bigger. 

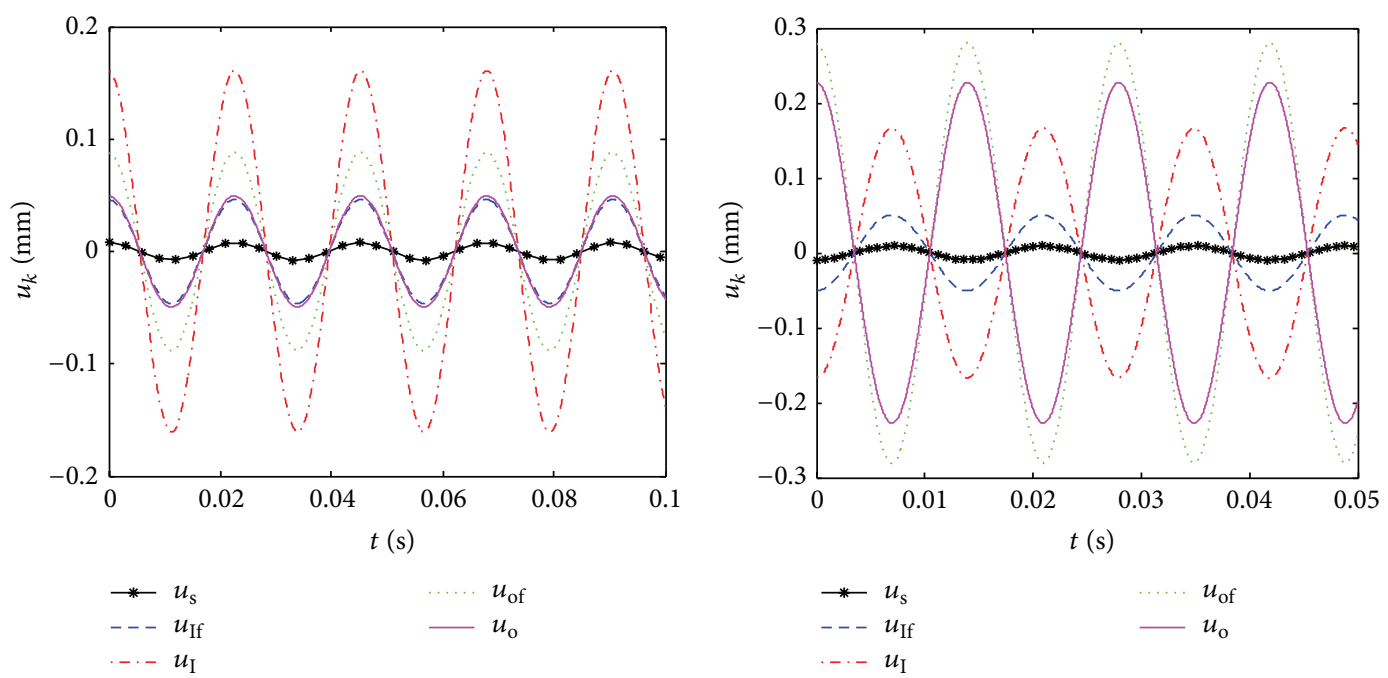

(a)

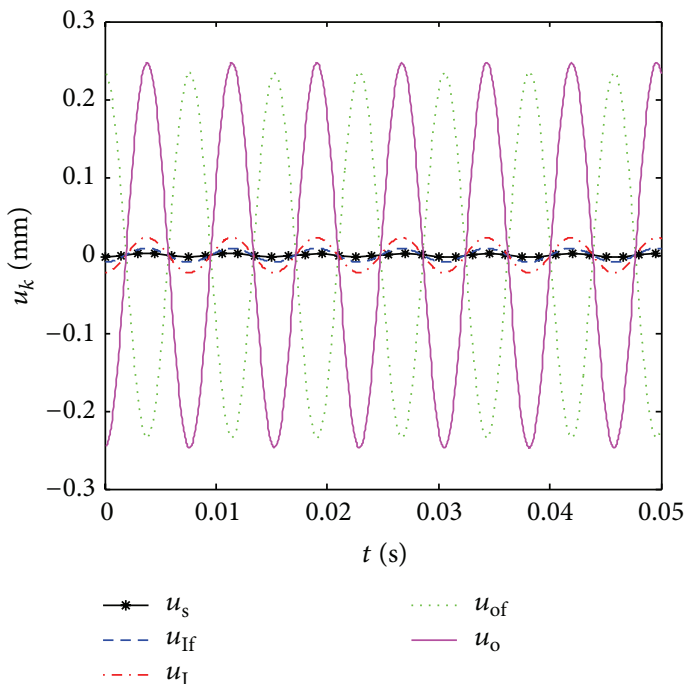

(c)

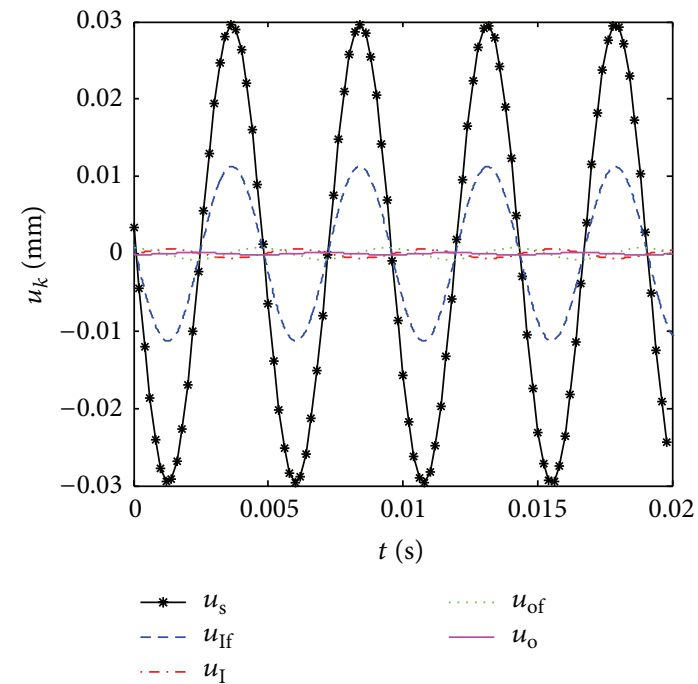

(d)

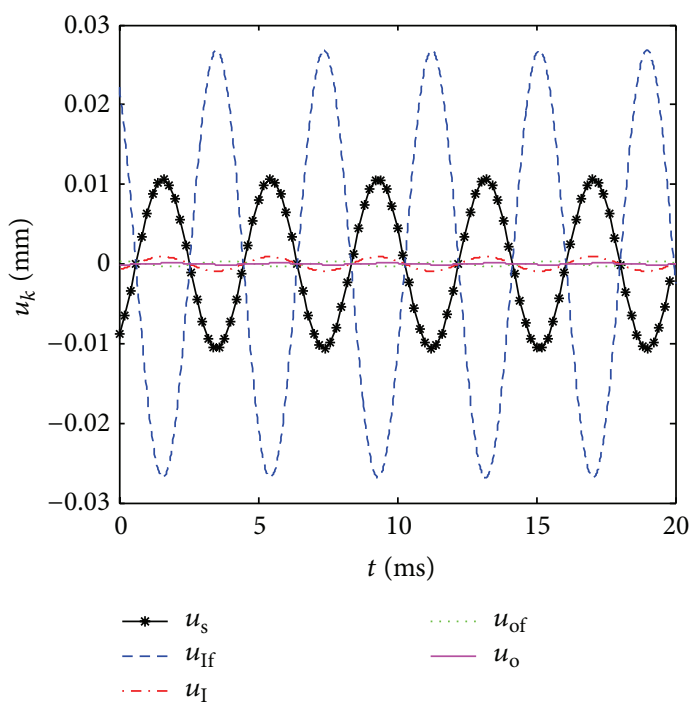

(e)

Figure 5: Main resonance of the EIMG system. 

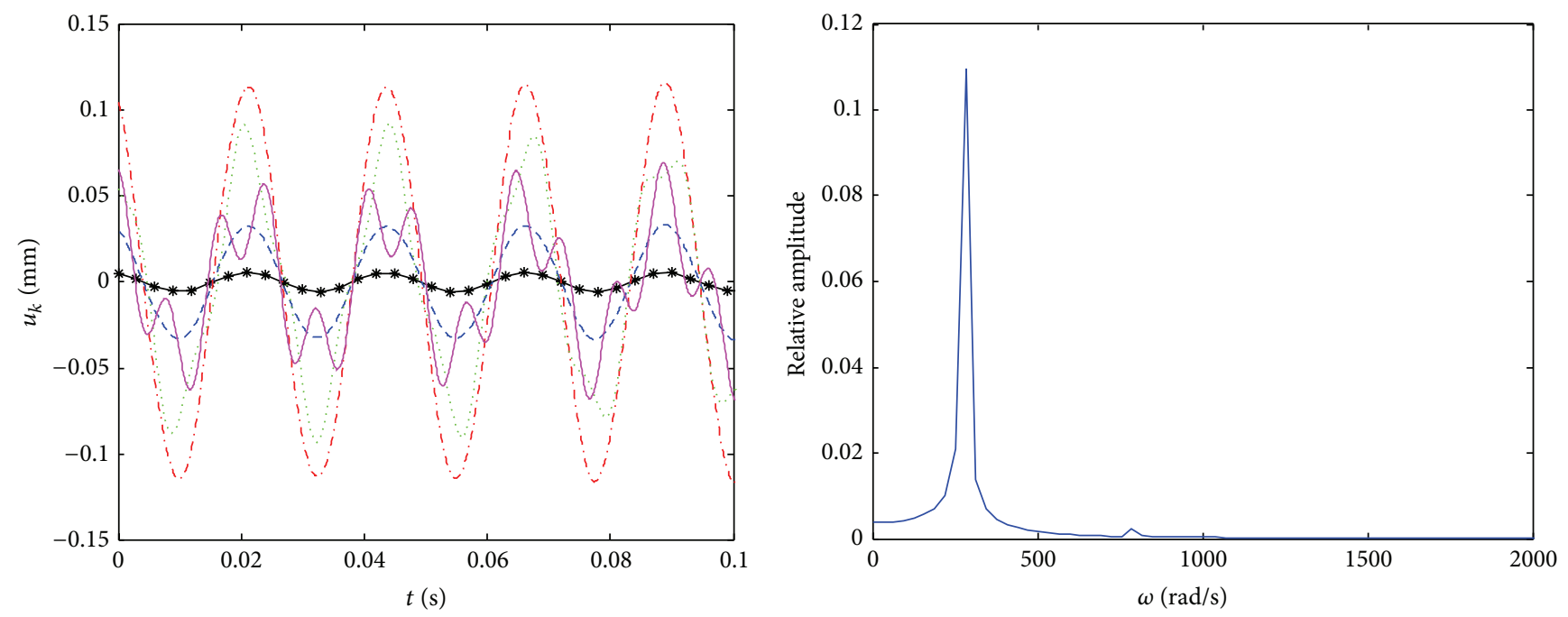

(a) $\omega_{\mathrm{e}} \approx \omega_{1}+\omega_{\mathrm{o}}$
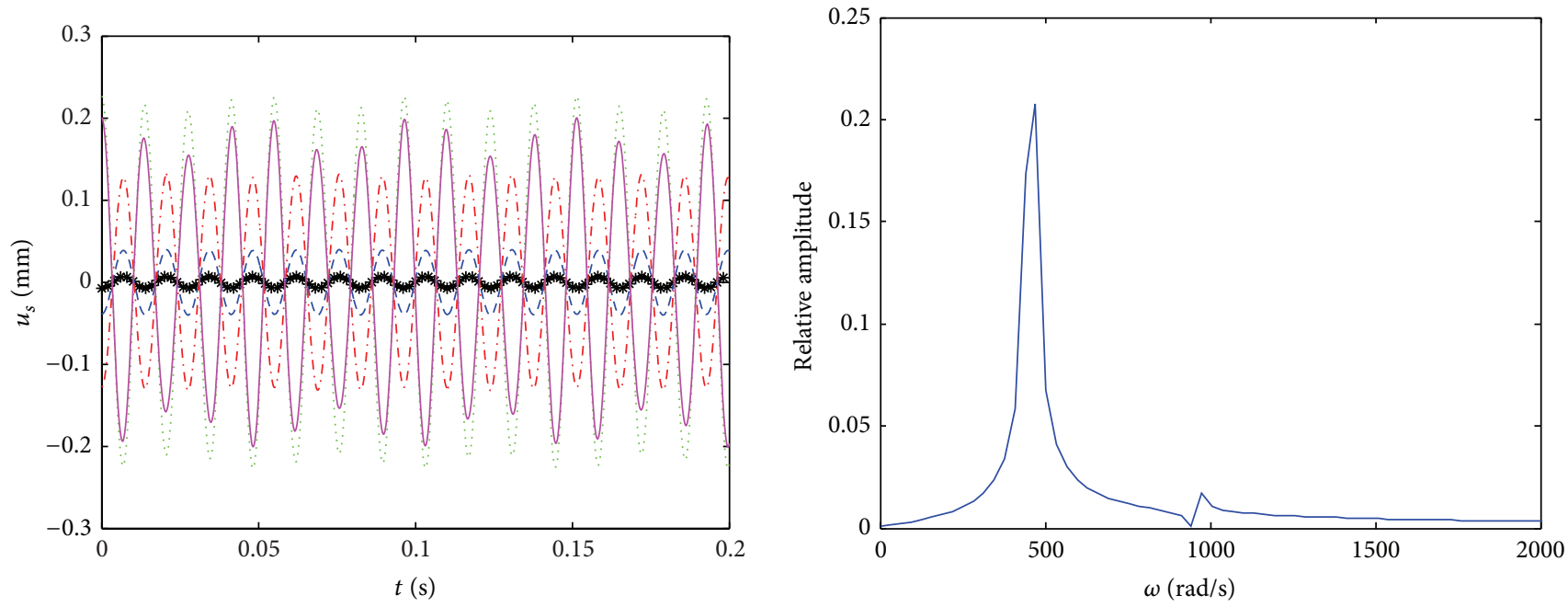

(b) $\omega_{\mathrm{e}} \approx \omega_{2}+\omega_{\mathrm{o}}$
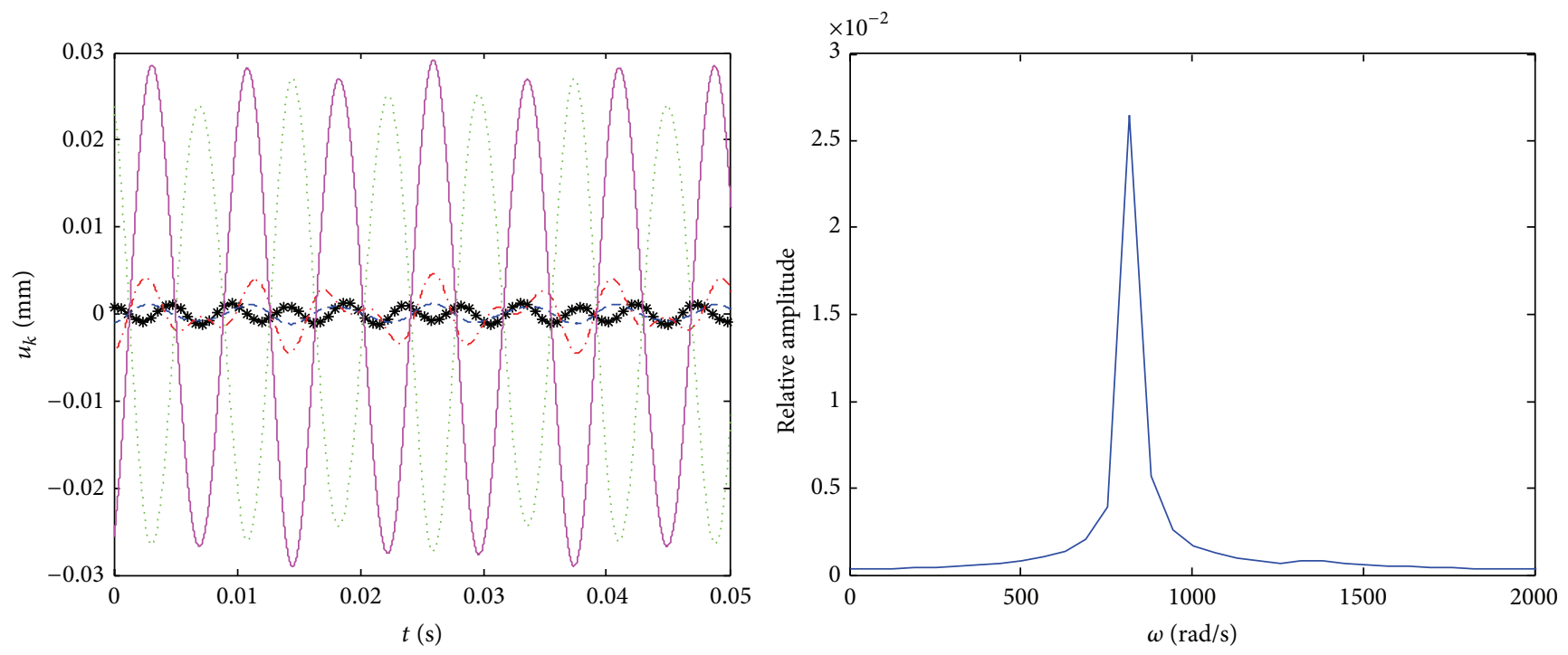

(c) $\omega_{\mathrm{e}} \approx \omega_{3}+\omega_{\mathrm{o}}$

Figure 6: Continued. 

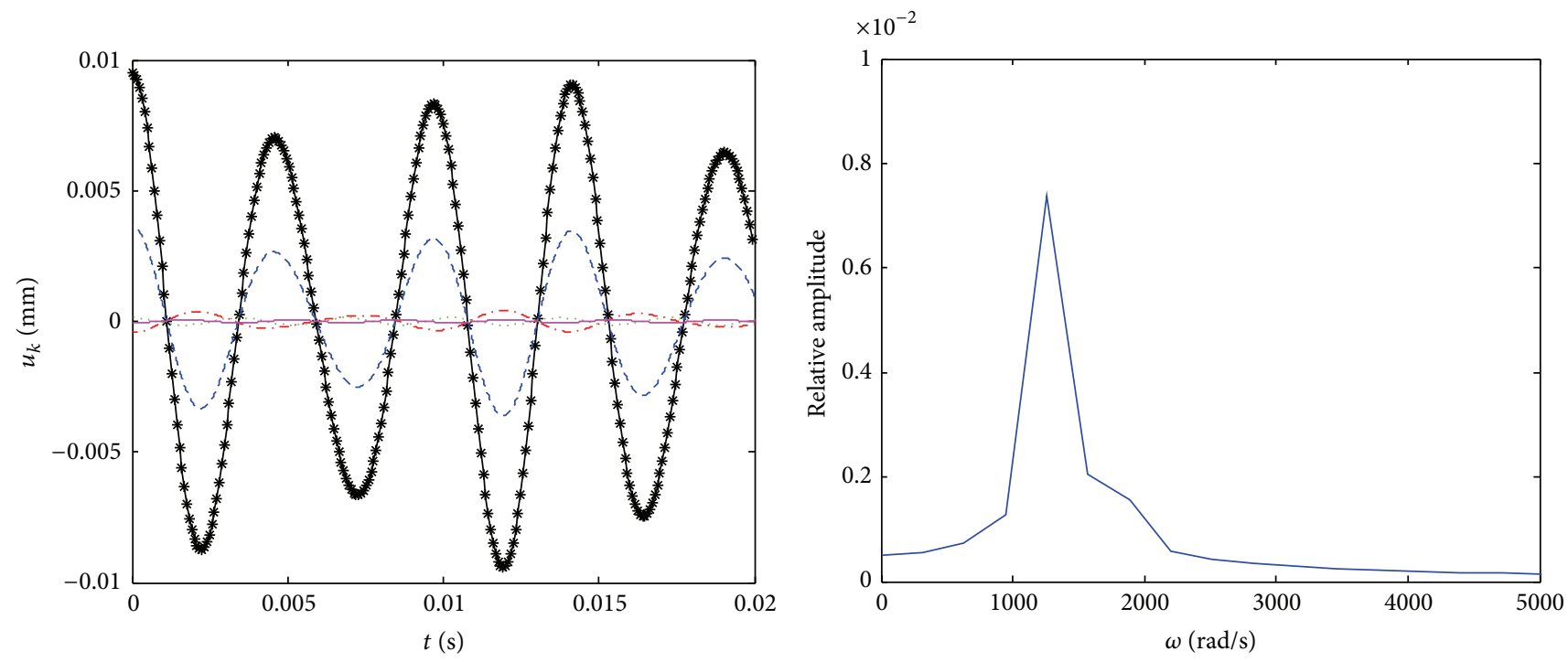

(d) $\omega_{\mathrm{e}} \approx \omega_{4}+\omega_{\mathrm{o}}$
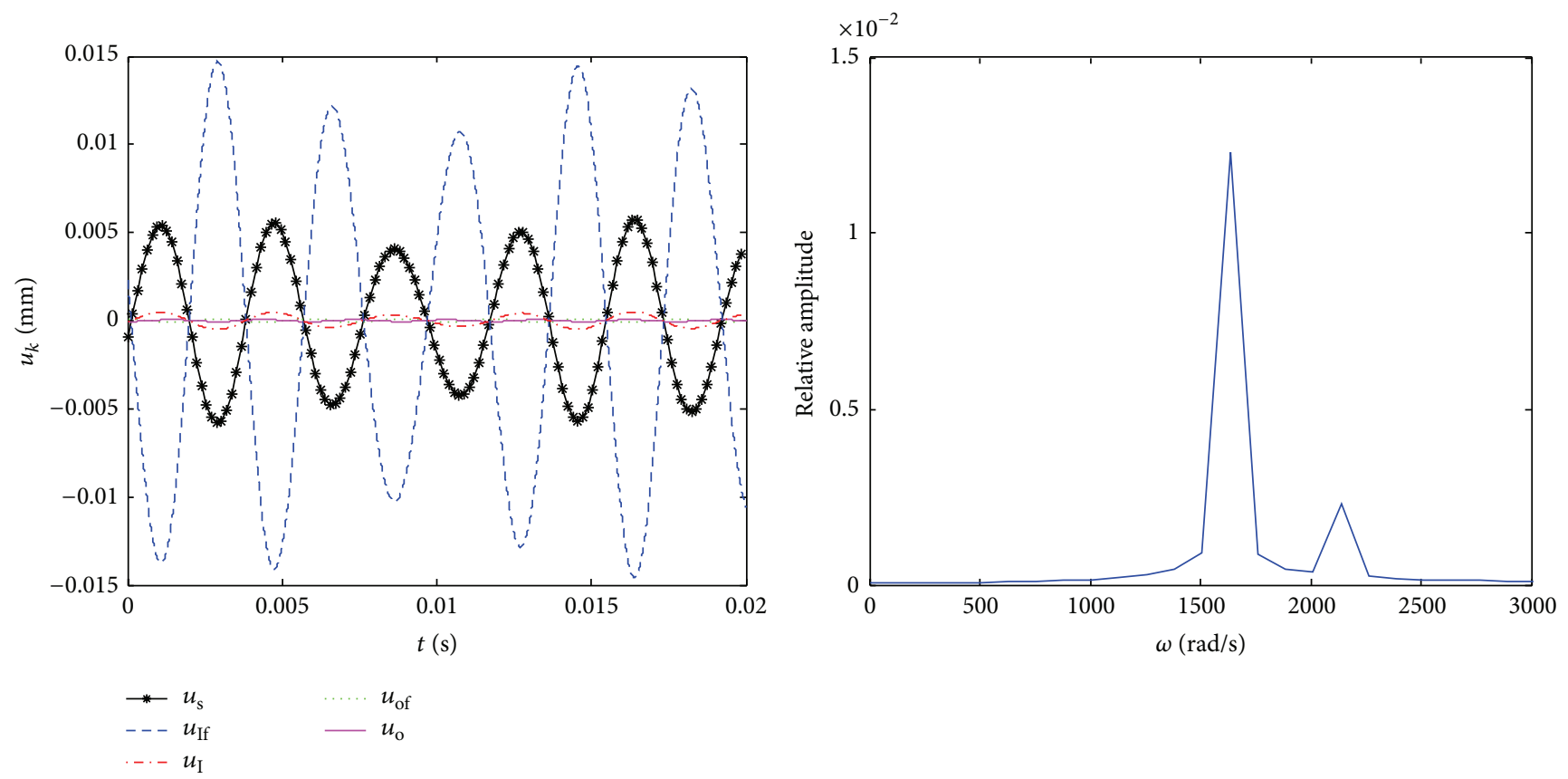

(e) $\omega_{\mathrm{e}} \approx \omega_{5}+\omega_{\mathrm{o}}$

FIGURE 6: Combination resonance of the EIMG system with $\omega_{\mathrm{e}} \approx \omega_{k}+\omega_{\mathrm{o}}$.

When the excited frequency is close to the combination frequencies and the natural frequencies, the mode energies will be motivated and the corresponding modal frequency will stand out. So the mode frequency, namely, the natural frequency, is the dominant frequency.

Combination resonances must be excited by the inner and outer exciting, and the value of the combination frequency is bigger. So, the combination resonances are not relatively easy to take place and the amplitudes of components are smaller. The resonance amplitudes caused by the combination exciting of the outer torque wave and the electromagnetic coupling stiffness between the inner stator and inner FP are smaller and are much smaller.
Considering other multiple harmonics components in the electromagnetic coupling stiffnesses, there are more resonance sources. In order to improve the dynamic characteristics, structure parameter optimizing and the dynamic parameter optimizing must be carried out.

\section{Conclusion}

Considering the magnetic field modulating, the electromagnetic coupling stiffnesses in EIMG system vary periodically and contain multiple harmonics, in which the main harmonic is the product of the angular frequency and the number of 

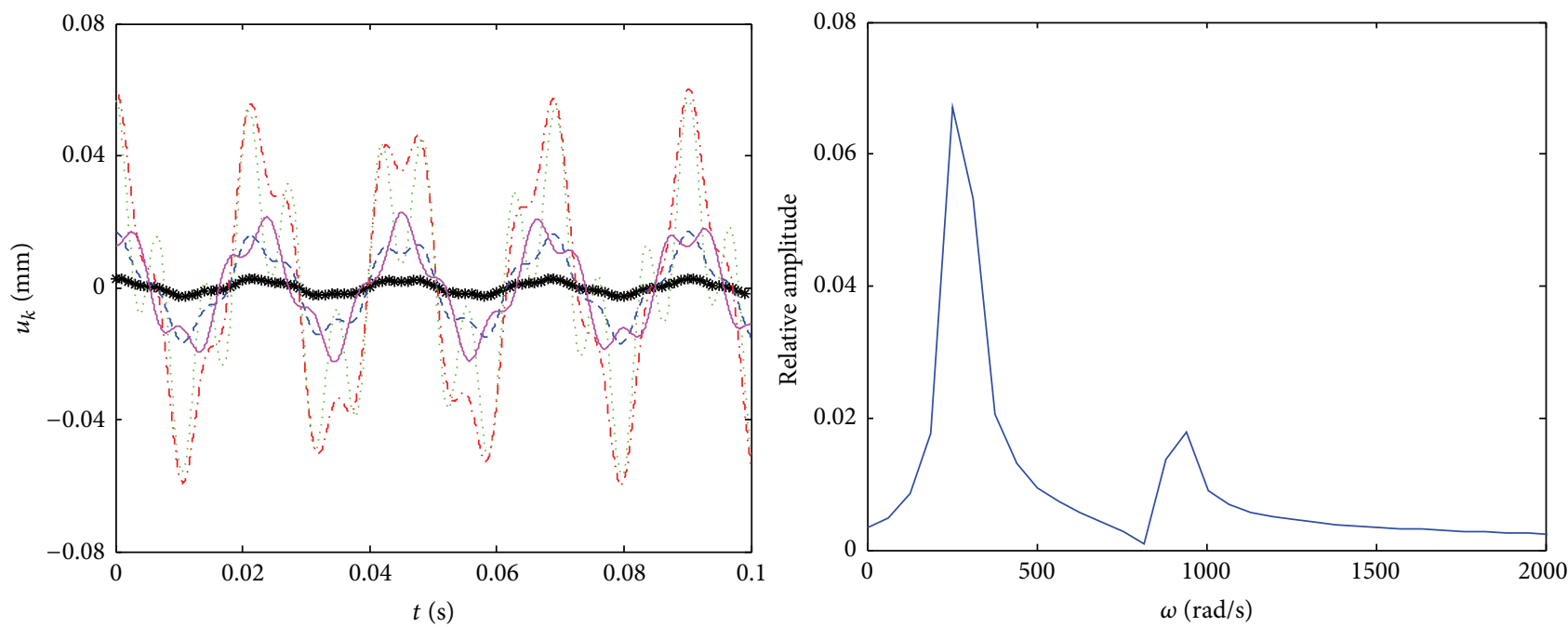

(a) $\omega_{\mathrm{e}} \approx \omega_{1}+\omega_{\mathrm{s}}$
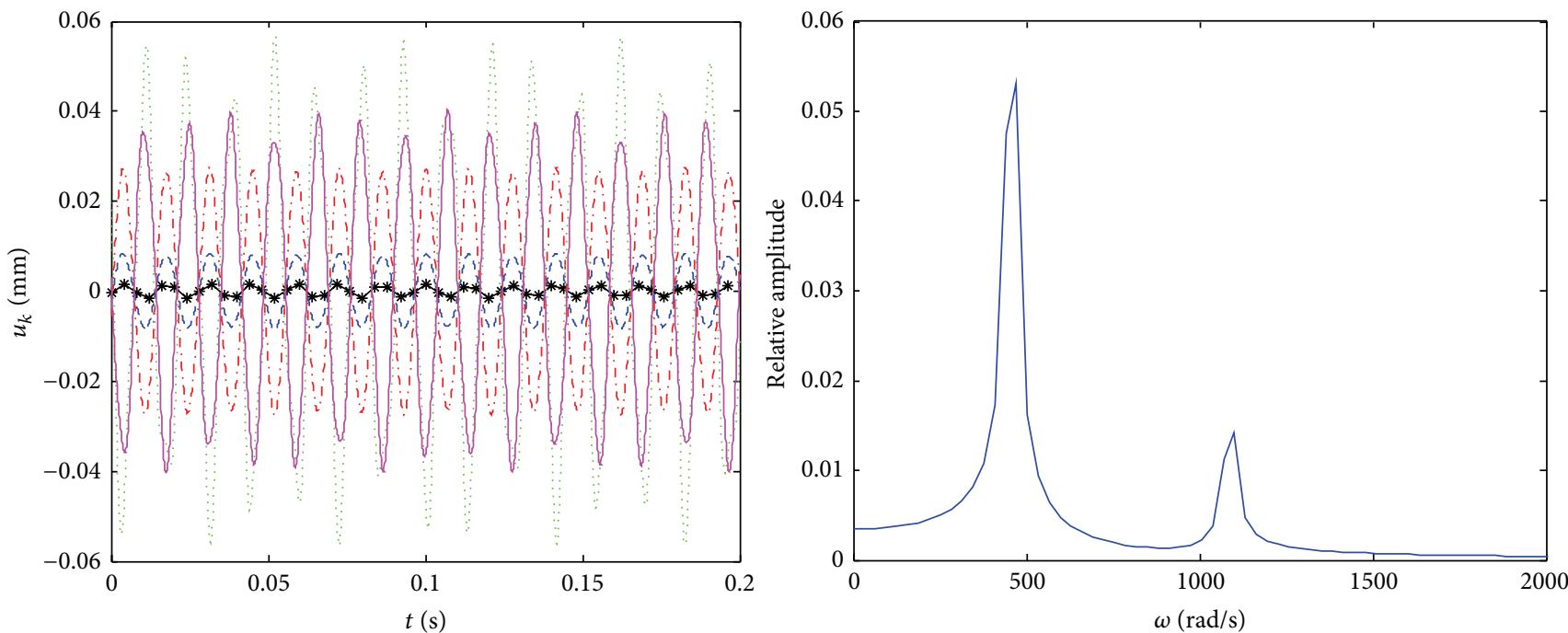

(b) $\omega_{\mathrm{e}} \approx \omega_{2}+\omega_{\mathrm{s}}$
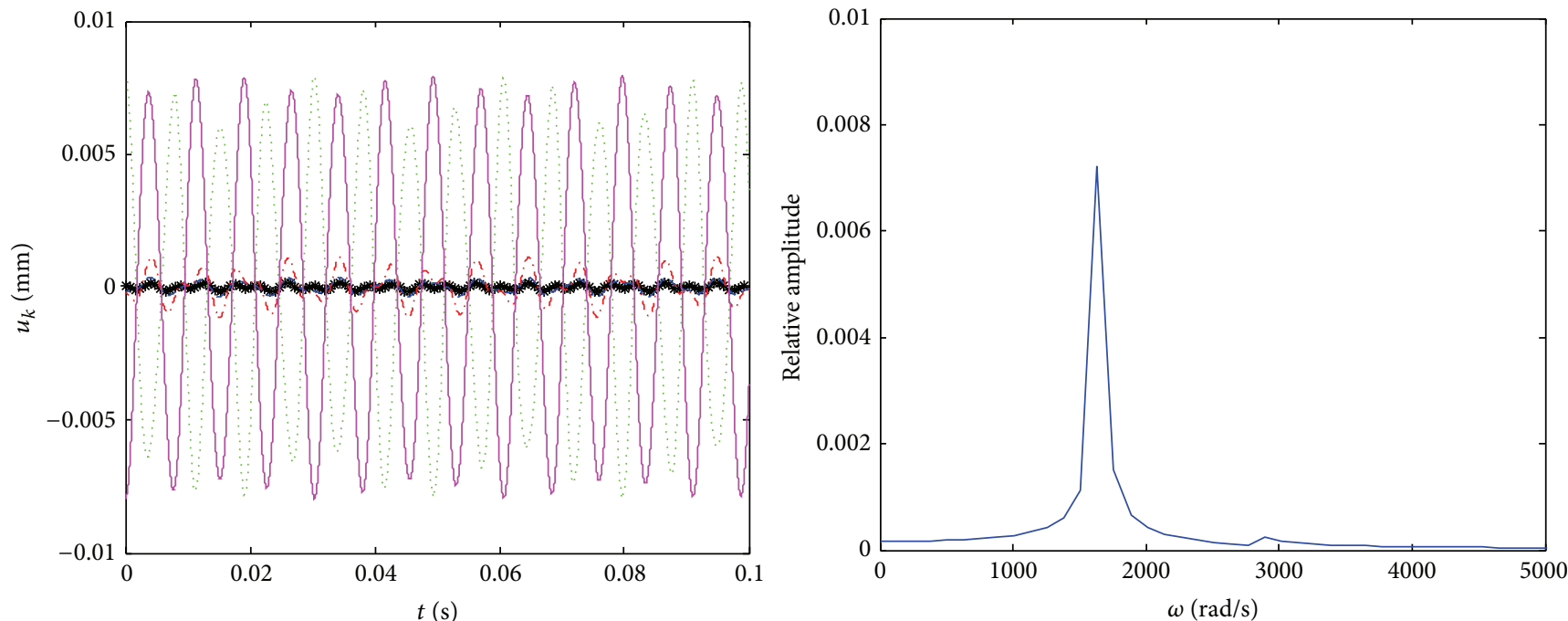

(c) $\omega_{\mathrm{e}} \approx \omega_{3}+\omega_{\mathrm{s}}$

Figure 7: Continued. 

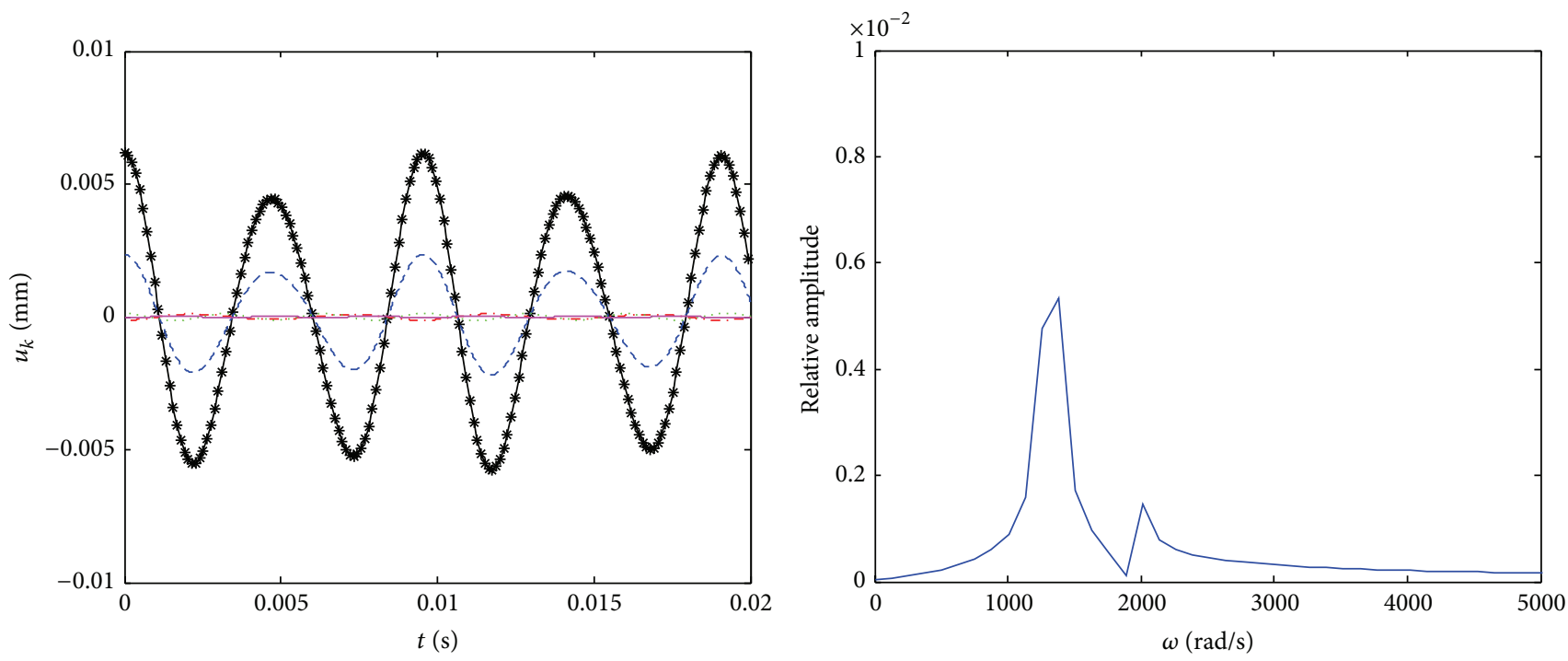

(d) $\omega_{\mathrm{e}} \approx \omega_{4}+\omega_{\mathrm{s}}$
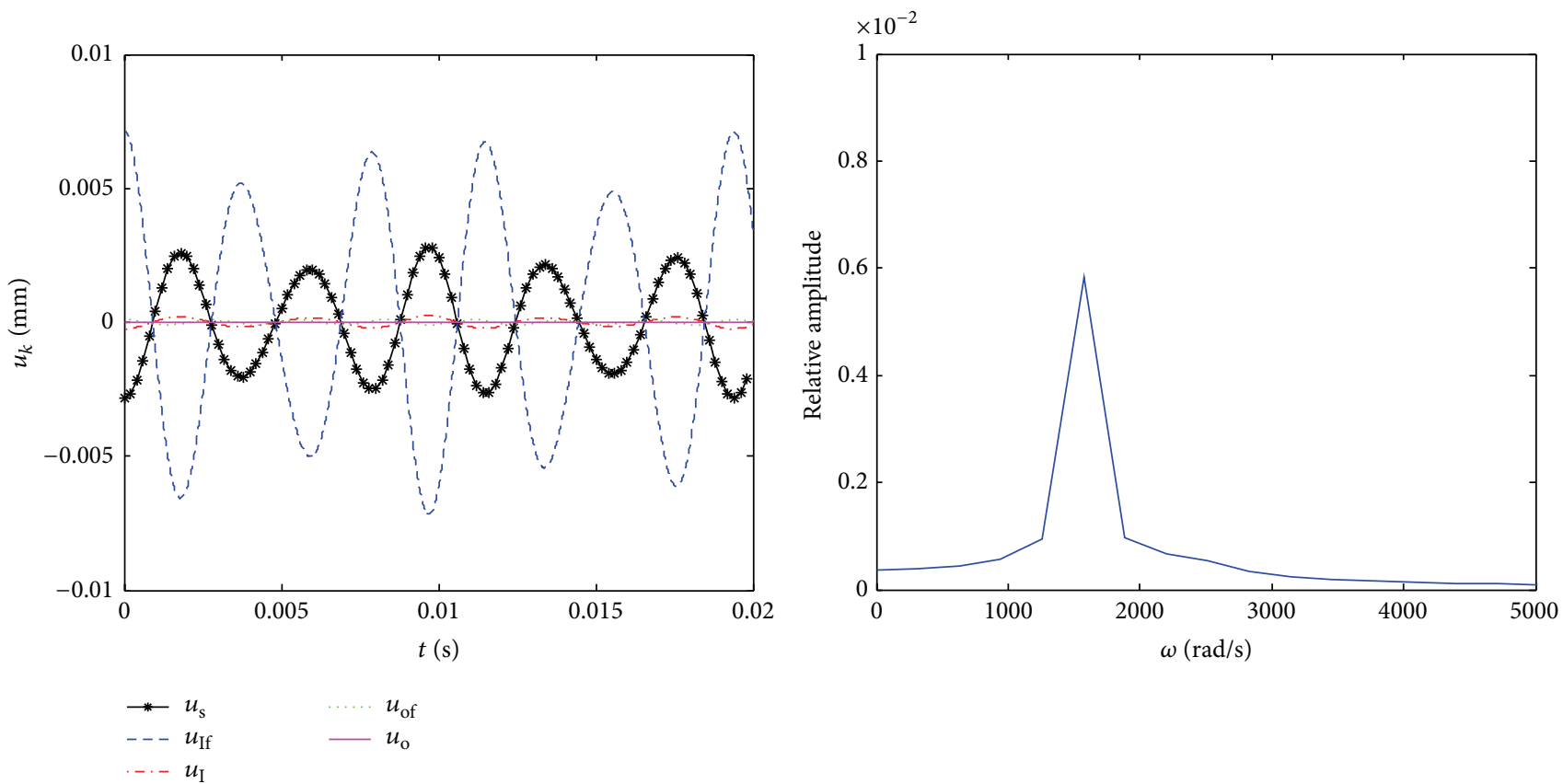

(e) $\omega_{\mathrm{e}} \approx \omega_{5}+\omega_{\mathrm{s}}$

Figure 7: Combination resonance of the EIMG system with $\omega_{\mathrm{e}} \approx \omega_{k}+\omega_{\mathrm{s}}$.

pole pairs. Considering the time-varying periodic stiffnesses, EIMG system is a typical parametric vibration system.

Considering the exciting on the outer FP and the timevarying electromagnetic coupling stiffnesses, there are the main resonances and the combination resonances in EIMG system. When the main resonances and the combination resonances occur, the dominant frequencies are always the natural frequency of EIMG system. When the exciting frequency increases from small to big, the rotational displacements of the inner rotor, the outer FP, the outer stator, the inner stator, and the inner FP will reach maxima.
The electromagnetic coupling stiffnesses are much smaller than the mechanical supporting stiffness. The relative resonance amplitudes of components have great difference. When the exciting frequency is close to the natural frequency of OFRM, IRRM, and OFRM, the resonances of EIMG system are bigger. The component amplitudes of the main resonances are much bigger than the components amplitudes of the combination resonances. Particularly, the meshing stiffness wave between the inner stator and the inner FP has little influence on EIMG system. 
The time-varying electromagnetic coupling stiffnesses make EIMG system be unstable. So the stability charts are the important research and must be discussed in next work.

\section{Conflict of Interests}

The authors declare that there is no conflict of interests regarding the publication of this paper.

\section{Acknowledgments}

This project is supported by Natural Science Foundation of China (51205341), Research Program of Natural Science at Universities of Hebei Province (Q2012032), and the Joint Fund of Specialized Research Fund for the Doctoral Program of Higher Education and Hebei Provincial Education Office (20121333120004).

\section{References}

[1] P. O. Rasmussen, T. O. Andersen, F. T. Jørgensen, and O. Nielsen, "Development of a high-performance magnetic gear," IEEE Transactions on Industry Applications, vol. 41, no. 3, pp. 764-770, 2005.

[2] K. Atallah and D. Howe, "A novel high-performance magnetic gear," IEEE Transactions on Magnetics, vol. 37, no. 4, pp. 28442846, 2001.

[3] K. Atallah, J. Wang, and D. Howe, "A high-performance linear magnetic gear," Journal of Applied Physics, vol. 97, no. 10, Article ID 10N516, 2005.

[4] L. Baghli, E. Gouda, S. Mezni, and A. Rezzoug, "Hybrid vehicle with a magnetic planetary gear," in Proceedings of the International Symposium on Environment Friendly Energies in Electrical Applications (EFEEA '10), Ghardaïa, Algeria, November 2010.

[5] N. W. Frank and H. A. Toliyat, "Gearing ratios of a magnetic gear for marine applications," in Proceedings of the IEEE Electric Ship Technologies Symposium (ESTS '09), pp. 477-481, Baltimore, Md, USA, April 2009.

[6] S. Mezani, K. Atallah, and D. Howe, "A high-performance axialfield magnetic gear," Journal of Applied Physics, vol. 99, no. 8, Article ID 08R303, 2006.

[7] L. Jian and K. T. Chau, "A coaxial magnetic gear with halbach permanent-magnet arrays," IEEE Transactions on Energy Conversion, vol. 25, no. 2, pp. 319-328, 2010.

[8] L. Jian, K. T. Chau, and J. Z. Jiang, "A magnetic-geared outerrotor permanent-magnet brushless machine for wind power generation," IEEE Transactions on Industry Applications, vol. 45, no. 3, pp. 954-962, 2009.

[9] D. J. Evans and Z. Q. Zhu, "Influence of design parameters on magnetic gear's torque capability," in Proceedings of the IEEE International Electric Machines and Drives Conference, pp. 14031408, Ontario, Canada, May 2011.

[10] L. Jian, G. Xu, J. Song, H. Xue, D. Zhao, and J. Liang, "Optimum design for improving modulating-effect of coaxial magnetic gear using response surface methodology and genetic algorithm," Progress in Electromagnetics Research, vol. 116, pp. 297-312, 2011.

[11] L. A. Percebon, R. Ferraz, and M. V. F. da Luz, "Modelling of a magnetic gear considering rotor eccentricity," in Proceedings of the IEEE International Electric Machines and Drives Conference (IEMDC '11), pp. 1237-1241, IEEE, Ontario, Canada, May 2011.
[12] X.-H. Hao, X.-M. Yuan, H.-F. Zhang, and L.-J. Zhang, "Combination resonances of parametric vibration system of the field modulated magnetic gear," Journal of Vibroengineering, vol. 16, no. 3, pp. 1590-1601, 2014.

[13] P. Velex and L. Flamand, "Dynamic response of planetary trains to mesh parametric excitations," Journal of Mechanical Design, vol. 118, no. 1, pp. 7-14, 1996. 

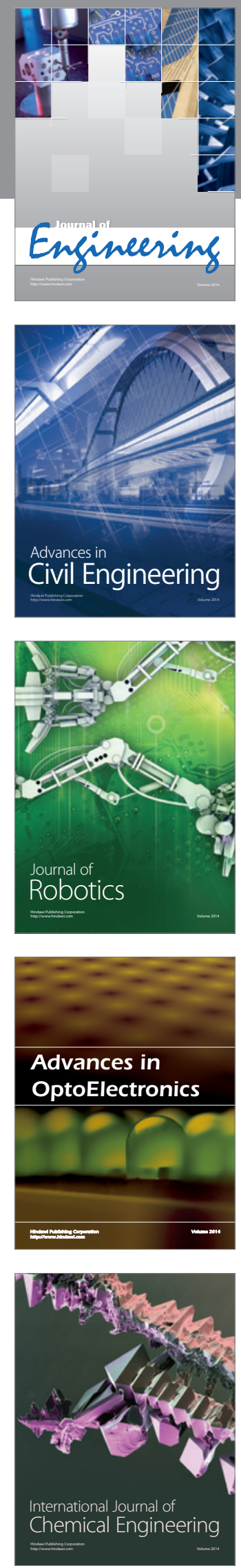

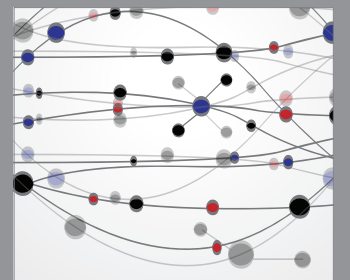

The Scientific World Journal
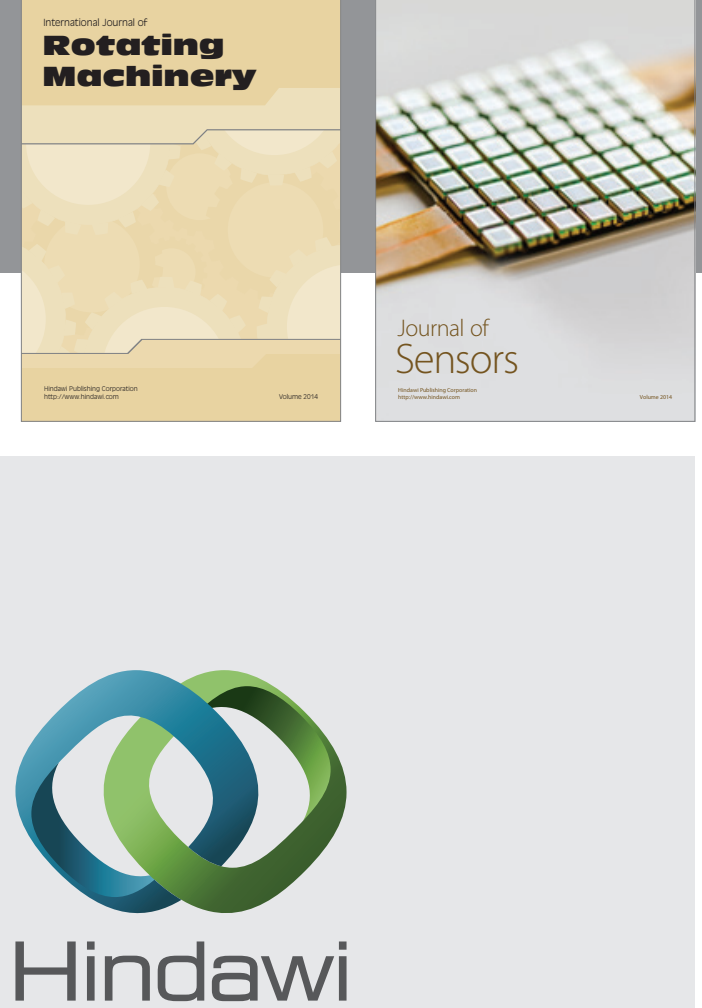

Submit your manuscripts at http://www.hindawi.com
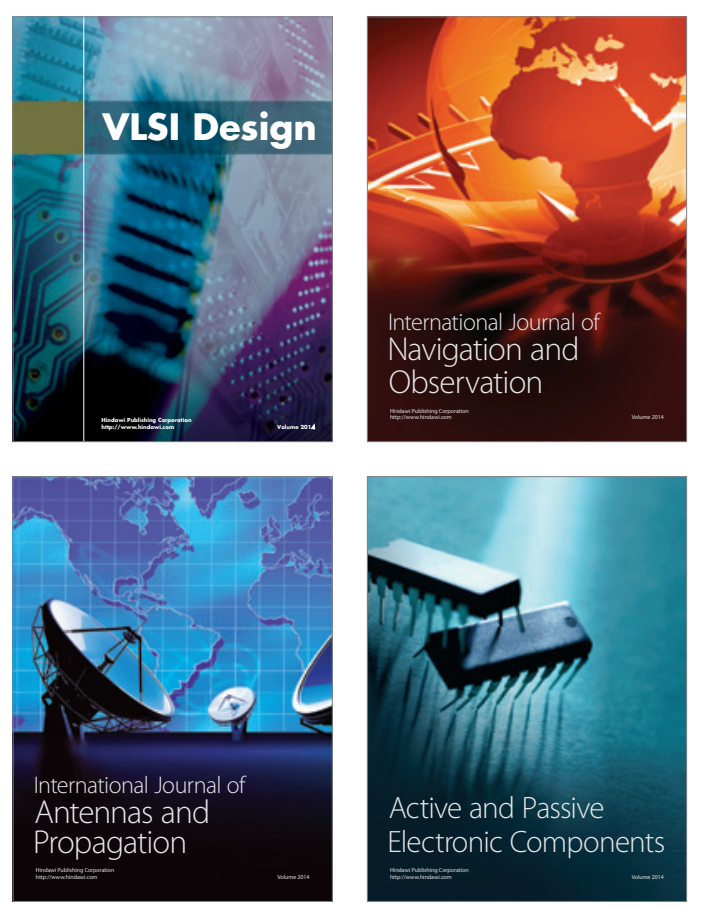
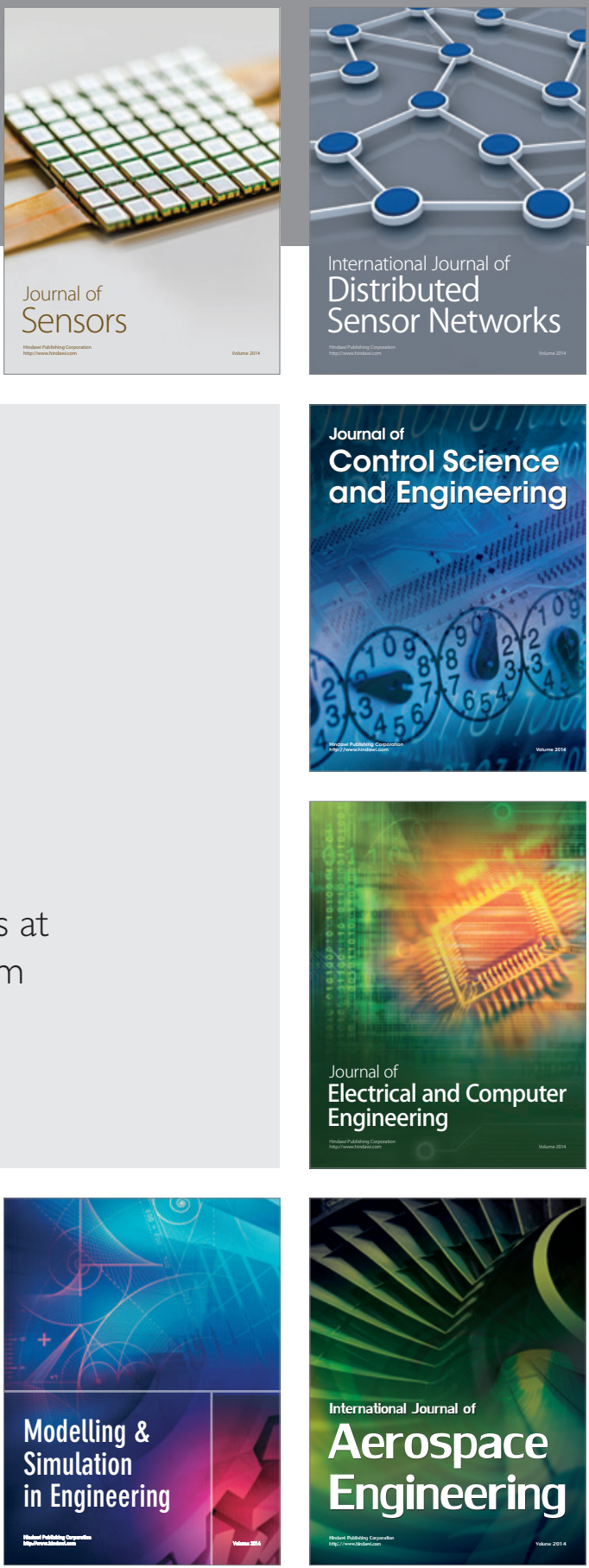

Journal of

Control Science

and Engineering
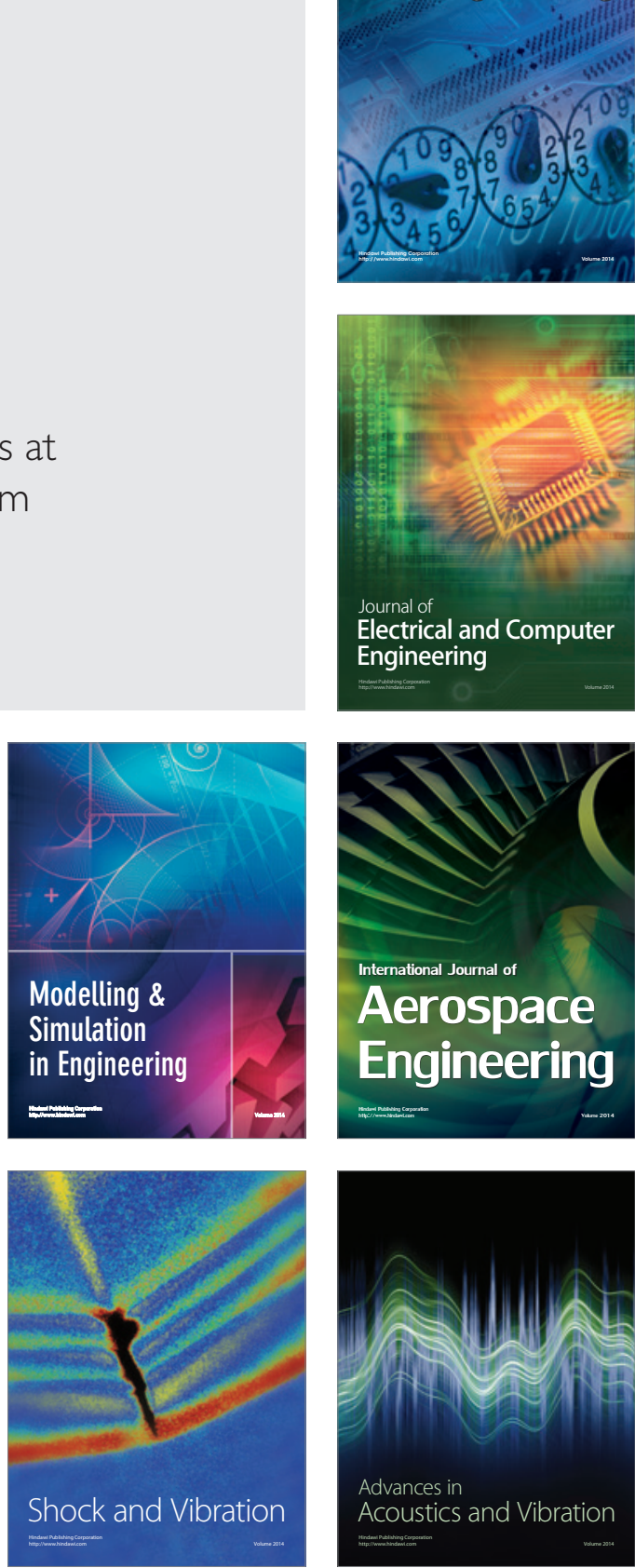